\title{
The origin of dust polarization in molecular outflows
}

\author{
S. Reiss $1^{1,2}$, D. Seifried ${ }^{3}$, S. Wolf ${ }^{2}$, R. Banerjee ${ }^{4}$, and R. S. Klessen ${ }^{1}$ \\ ${ }^{1}$ University of Heidelberg, Institute of Theoretical Astrophysics, Albert-Ueberle-Str. 2 U04, 69120 Heidelberg, Germany \\ e-mail: [reissl;klessen] @uni-heidelberg.de \\ 2 Institut für Theoretische Physik und Astrophysik, Christian-Albrechts-Universität zu Kiel, Leibnizstraße 15, 24098 Kiel, Germany \\ e-mail: sreissl@astrophysik.uni-kiel.de \\ 3 I. Physikalisches Institut, Universität zu Köln, Zülpicher Straße 77, 50937 Köln, Germany \\ ${ }^{4}$ Hamburger Sternwarte, Universität Hamburg, Gojenbergsweg 112, 21029 Hamburg, Germany
}

Received 8 January 2017 / Accepted 7 March 2017

\begin{abstract}
Aims. Polarization measurements of dust grains aligned with the magnetic field direction are an established technique for tracing largescale field structures. In this paper we present a case study to investigate the conditions that need to be met to detect a characteristic magnetic field substructure that is embedded in such a large-scale field. A helical magnetic field with a surrounding hourglass-shaped field is expected from theoretical predictions and self-consistent magnetohydrodynamical (MHD) simulations to be present in the specific case of protostellar outflows. Hence, such an outflow environment is the perfect environment for our study.

Methods. We present synthetic polarization maps in the infrared and millimeter regime of simulations of protostellar outflows. The simulations were performed with the newly developed radiative transfer and polarization code POLARIS. The code is the first to include a self-consistent description of various alignment mechanisms such as the imperfect Davis-Greenstein (IDG) and the radiative torque (RAT) alignment. We investigated the effects of the grain size distribution, inclination, and applied alignment mechanism.

Results. We find that the IDG mechanism cannot produce any measurable polarization degree $(\geq 1 \%)$, whereas the RAT alignment produced polarization degrees of a few percent. Furthermore, we developed a method for identifying the origin of the polarization. We show that the helical magnetic field in the outflow can only be observed close to the outflow axis and at its tip, whereas in the surrounding regions the hourglass field in the foreground dominates the polarization. Furthermore, the polarization degree in the outflow lobe is lower than in the surroundings, in agreement with observations. We also find that the orientation of the polarization vector flips around at about a few hundred micrometers because of the transition from dichroic extinction to thermal re-emission. In order to avoid ambiguities when interpreting polarization data, we therefore suggest to observe in the far-infrared and millimeter regime. The actual grain size distribution has only little effect on the emerging polarization maps. Finally, we show that it is possible to observe the polarized radiation emerging from protostellar outflows with ALMA.
\end{abstract}

Key words. radiative transfer - galaxies: ISM - ISM: jets and outflows - methods: numerical - techniques: polarimetric

\section{Introduction}

Magnetic fields are responsible for protosteller outflows, which are one of the most prominent signs of star formation (Pudritz \& Norman 1983). These outflows are more easy to detect than the embedded protostellar disks from where they are expected to be emitted. Hence, the observation of an outflow is a strong indicator for the presence of a rotating disk. Moreover, the launching mechanism of the outflow is closely linked to the disk rotation since the magnetic field lines are frozen at the disk scale and are dragged along the direction of rotation. The magnetic field morphology in this scenario is expected to have a strong toroidal component (e.g., Blandford \& Payne 1982; Pudritz \& Norman 1983; Shibata \& Uchida 1985; Tomisaka 1998; Banerjee et al. 2006) that forms a helical field morphology together with the large-scale poloidal field of the surrounding medium. The detection of a helical field component in an outflow would accordingly be an additional indicator for the presence of a rotating disk that is embedded in the center.

From an observational point of view it needs to be verified by proper modeling whether the helical field morphological would be detectable in the interior of outflow lobes or if the surrounding hourglass field dominates light polarization. While recent observations of $\mathrm{CO}$ polarization measurements seem to confirm the hypothesis of a helical field (Ching et al. 2016), the interpretation of such data remains still unclear since the magnetic field morphology in the ambient environment represents a possible source of ambiguity. The large-scale magnetic field is expected to be hourglass-shaped and is therefore orientated perpendicular to the toroidal component along the line of sight (LOS), which hides the embedded helical field morphology (e.g., Girart et al. 2006, 2009).

By focusing on the aspect of the magnetic morphology, this can be probed by dust polarization measurements. Historically, the alignment of non-spherical dust grains has long been suggested as an explanation for the observed polarization of stellar radiation (Hiltner 1949; Hall 1949; Martin 1971).

Different theories of grain alignment agree that rotating nonspherical dust grains tend to align with the magnetic field direction (see Lazarian 2007). Unpolarized light will gain polarization by dichroic extinction in the mid-infrared (mid-IR) and thermal dust re-emission from far-infrared (far-IR) to submillimeter (submm) and millimeter ( $\mathrm{mm}$ ) wavelength (e.g., Frau et al. 2011), which precludes inferring the projected magnetic field 
morphology. Polarization measurements therefore provide a promising tool to determine the morphology and subsequently to investigate of the role of magnetic fields in the evolution (Hildebrand et al. 2000; Crutcher 2004; Girart et al. 2012) of star-forming systems. The difficulty is that the reliability of polarization measurements and their interpretation depends on a wide range of physical parameters that are still discussed (see Andersson et al. 2015, for a review).

With dedicated instruments such as the Atacama Large Millimeter Array (ALMA, Brown et al. 2004) and the highresolution Airborne Wideband Camera-plus instrument of the airborne Stratospheric Observatory For Infrared Astronomy (HAWC+/SOFIA, Dowell et al. 2013), the measurement of dust polarization of cloud cores and disks potentially becomes feasible, and in turn, questions about the potential of multiwavelength polarization measurements to identify specific components of larger structures with complex magnetic field morphology may be answered.

Several observations measuring the magnetic field of molecular outflows and the magnetic field in the center of molecular cloud cores are currently available. The results, however, seem to contradict each other. Whereas Davidson et al. (2011) and Chapman et al. (2013) report magnetic fields that are preferentially aligned with outflows, Hull et al. $(2013,2014)$ find magnetic fields to be strongly misaligned with respect to the outflow axis. Hence, simulations of synthetic observations are essential to asses to what accuracy the structure of magnetic fields in starforming cores can be inferred from observations. Even for a well-defined field structure as presented in Reissl et al. (2014), the resulting polarization pattern is extremely complex and thus not easy to interpret. This holds even more when turbulent motions are involved. We therefore argue that possible conclusions drawn from such observations have to be considered with great care.

The problems tied to the correct interpretation of dust polarization observations that need to be addressed are listed below.

- The measured polarization naturally is only be a projection of the underlying magnetic field morphology since it is averaged along a particular LOS. The question remains to what extend - if at all - the 3D structure of the underlying magnetic field can be deduced by dust polarization measurements.

- It is not clear a priori whether the polarization is observed in dichroic extinction or re-emission since these two competing mechanisms are both at work simultaneously. Whether the projected magnetic field is perpendicular or parallel to the measured polarization vector is an often neglected ambiguity in the literature.

- The observed degree of polarization strongly depends on the composition and size of the dust grains. This produces uncertainties that emerge from unconstrained dust properties, in particular in dense star-forming regions.

- Different theories can account for the alignment of dust grains under certain conditions. Each dust alignment theory comes with its characteristic polarization pattern, making the analysis of polarization measurements highly dependent on the choice of the considered theory.

In order to claim a correlation between the observed orientation of linear polarization and a particular magnetic field structure, careful RT modeling is required to ensure that the observed wavelengths are suitable for probing the regions of interest with aligned dust grains in the first place. However, for RT simulation modeling dust polarization maps, often only the density-weighted magnetic field is summed along the LOS (e.g., Soler et al. 2013) or the degree of polarization is adjusted to match observational data (Padovani et al. 2012). Thus, these attempts are of limited predictive capability since they oversimplify the complex physics of radiation-dust interaction and dust grain alignment physics.

For this reason, fully self-consistent radiative transfer simulations on linear polarization of radiation incorporating dust grain composition and a well-motivated grain alignment efficiency are required. To meet this requirement, we made use of the newly developed 3D RT code POLARIS (Reissl et al. 2016), the only code currently available that is capable of polarization calculations on non-spherical partially aligned dust grains. We study the polarization of mid-IR to $\mathrm{mm}$ radiation that is due to aligned dust grains. Here, we present a first analysis of synthetic polarization observations obtained from a post-processed magnetohydrodynamical (MHD) simulation (see Seifried et al. 2011, 2012). The simulation models the self-consistent formation of a protostellar disk and its associated molecular outflow driven in the Class 0 stage.

The structure of the paper is as follows: first, we present the properties of the chosen MHD simulation in Sect. 2. We then show details of the applied dust model in Sect. 3. In Sect. 4 the considered theories of dust grain alignment are introduced, followed by the description of the RT techniques in Sect. 5. We show the synthetic intensity and polarization maps as a function of different RT simulation parameters in Sect. 6. In Sect. 7 we present a method for determining the origin of polarization by means of tracing distinct rays in RT calculations. We take the influence of dust grain size on polarization into account in Sect. 8 and simulate ALMA polarization observations in Sect. 9. Finally, we discuss and summarize the results in Sects. 10 and 11, respectively.

\section{MHD outflow simulation}

The MHD simulation considered in this paper results in the formation of protostars, their surrounding disk, and the protostaller outflow following the collapse of the parental protostellar core. These simulations were performed with the astrophysical code FLASH (Fryxell et al. 2000) using the reliable MHD solver developed by Bouchut et al. (2007). To follow the long-term evolution of the protostellar disk and its associated outflow, we made use of sink particles (Federrath et al. 2010). For more details on the numerical methods, we refer to Seifried et al. $(2011,2012)$.

We analyze the results of a particular simulation considering the collapse of a $100 M_{\odot}$ molecular cloud core without initial turbulence, which is $0.25 \mathrm{pc}$ in diameter and initially rotating rigidly around the $z$-axis with a rotation frequency of $3.16 \times 10^{-13} \mathrm{~s}^{-1}$. The ratio of rotational to gravitational energy is $\beta_{\text {rot }}=4 \times 10^{-2}$. The magnetic field is initially aligned with the rotation axis, that is, it is parallel to the $z$-axis and has a strength chosen such that the normalized mass-to-flux ratio is (Spitzer 1978)

$\mu=\left(\frac{M_{\text {core }}}{\Phi_{\text {core }}}\right) /\left(\frac{M}{\Phi}\right)_{\text {crit }}=\left(\frac{M_{\text {core }}}{\int B_{z} \mathrm{~d} A}\right) /\left(\frac{0.13}{\sqrt{G}}\right)=26$.

This corresponds to an initial central magnetic field strength of $132 \mu \mathrm{G}$. Hence, the core is magnetically supercritical and the magnetic field cannot significantly counteract the initial gravitational collapse of the core. Again, we refer to (Seifried et al. 2011, 2012) the simulation is called 26-4 within these papers for more details on the initial conditions. 

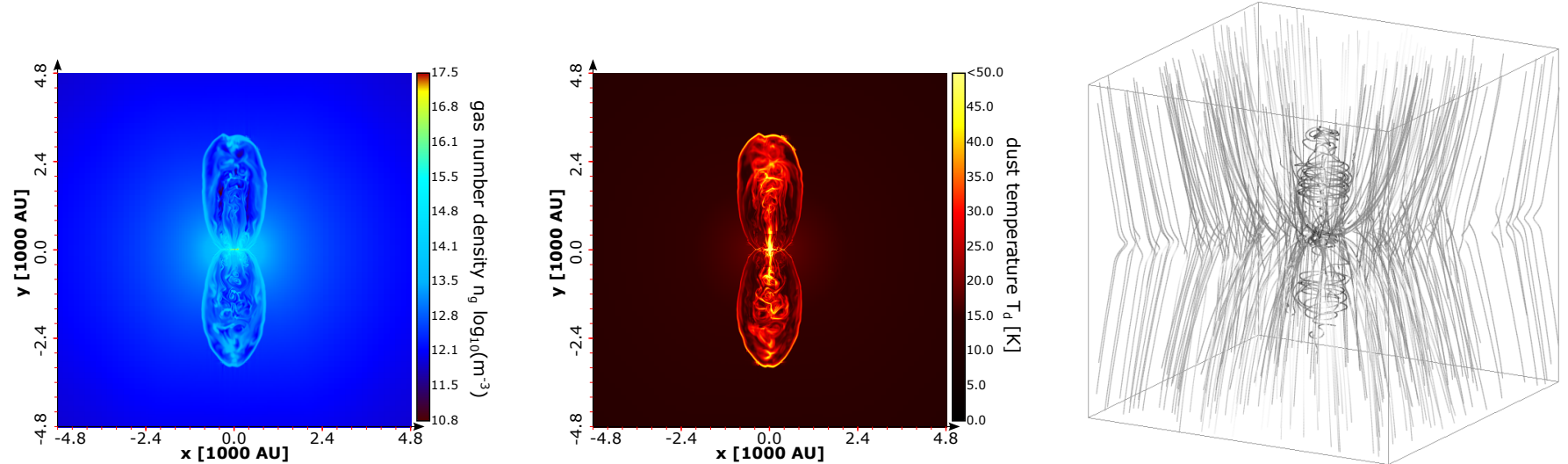

Fig. 1. Left panel: gas number density $n_{\mathrm{g}}$ of the considered MHD simulation in the mid-plane parallel to the symmetry axis of the outflow lobes. Middle panel: corresponding dust temperature $T_{\mathrm{g}}$. The dust temperature was post-processed to account for the stellar contribution of the protostars to dust heating (see Sect. 4 for details). Right panel: 3D magnetic field distribution. The characteristic helical component is in the interior of the outflow lobes that are embedded within a large-scale hourglass-shaped field. The box has edge lengths of 9200 AU.

During the collapse of the core, a rotationally supported disk builds up around the first protostar. The disk starts to fragment after $\sim 2600 \mathrm{yr}$, forming a small cluster of six protostars in total. A magneto-centrifugally driven protostellar outflow is launched after the formation of the first sink particle, which is well collimated with a collimation factor of $\sim 4$ at the end of the simulation. The outflow continues to expand in a roughly self-similar fashion and maintains the overall morphological properties.

We analyzed this outflow at an age of $5000 \mathrm{yr}$. By this time, the outflow lobe has a height of $3200 \mathrm{AU}$ above and below the disk midplane and a maximum outflow speed of about $19 \mathrm{~km} \mathrm{~s}^{-1}$, which is well above the escape velocity. We note that this maximum value is attained close to the disk and that most of the gas in the outflow is a few $\mathrm{km} \mathrm{s}^{-1}$ slower. The six protostars cover a range of luminosities between $0.22 L_{\odot}$ and $81.4 L_{\odot}$. We model the luminosities and also take the accretion onto the surface of the protostars into account, which provides a significant part of the total luminosity at this stage of star formation (see Offner et al. 2009, for details).

The mid-plane gas number density $n_{\mathrm{g}}$, dust temperature $T_{\mathrm{d}}$, and the 3D magnetic field morphology of the considered snapshot are shown in Fig. 1. Investigating the magnetic field structure within the outflow lobes, we find that the toroidal magnetic field component clearly dominates the poloidal component, while the field outside the outflow lobe is hourglass shaped. Furthermore, we observed the occurrence of several shocks within the outflow, which locally lead to rather chaotic gas motions and magnetic field orientations.

\section{Constraints to dust modeling}

The interpretation of observational polarization data strongly depends on the parameter of the considered dust grain model. While we know that grains in the interstellar medium (ISM) are not spherically symmetric, their actual shape, composition, and size distribution is uncertain.

A satisfactory model, the so-called MRN model (Mathis et al. 1977), that reproduces the galactic extinction curve is a three-parameter model with a power-law size distribution $n(a) \propto a^{-q}$ and a dust grain size range of $a \in\left[a_{\min }: a_{\max }\right]$ with values of $q=-3.5, a_{\min }=5 \mathrm{~nm}$, and $a_{\max }=250 \mathrm{~nm}$. In subsequent studies, the upper limit was extended to be of $\mu \mathrm{m}$-size (e.g., Clayton et al. 2003; Draine \& Li 2007). To account for characteristic extinction and absorption features, an ensemble of dust materials is used that consists of a mixture of carbonaceous (graphite) and silicate (olivene) materials (e.g., Zubko 1995; Zhukovska et al. 2016). Additionally, in the case of IDG alignment, we considered ferromagnetic particles encapsulated in the dust grains, which enhances the paramagnetic alignment by a factor of $10^{2}$ (Jones \& Spitzer 1967; Djouadi et al. 2007; Belley et al. 2009). An oblate dust grain with an fixed aspect ratio represents a particularly promising approach for simulating the interstellar polarization and extinction data (Lee \& Draine 1985; Kim \& Martin 1995; Hildebrand \& Dragovan 1995), therefore we use an average value of 0.5 .

Since the different grain materials have unique dielectric and paramagnetic properties, this also results in a unique alignment behavior. Analyzing the linear polarization and circular polarization shows that a higher alignment efficiency is to be expected for silicate grains, while carbonaceous grains seem to remain unaffected by a magnetic field (e.g., Martin \& Angel 1976; Mathis 1986). We therefore considered carbonaceous grains to be randomized, while silicate grains are partially aligned in our model (Clayton et al. 2003; Draine \& Li 2007).

The dust properties required for the self-consistent dust grain heating calculations and polarization simulations are the efficiencies $Q$ of light polarization parallel $\left(Q_{\|}\right)$and perpendicu$\operatorname{lar}\left(Q_{\perp}\right)$ to the grain symmetry axis (see e.g., Martin 1971). The efficiencies can be precalculated by a numerical method representing the dust grain shapes as an array of material specific discrete dipoles (Draine \& Flatau 2000). In order to remain consistent with the constraints of the interstellar dust parameter, we used the DDSCAT 7.2 code (see Draine \& Flatau 2013) to calculate values of $Q$ for an aspect ratio of 0.5 in a regime of wavelength $\lambda \in[0.9 \mu \mathrm{m}: 3 \mathrm{~mm}]$ and grain sizes limits of $a_{\min }=5 \mathrm{~nm}$ and $a_{\max }=2 \mu \mathrm{m}$. Here, the number of dipoles in our calculations ranges from $N=171500-296352$ to remain in the numerical limit $(a<0.05 \lambda \sqrt[3]{N} /|m|)$ of the DDSCAT code, where $|m|$ is the complex refractive index. For the input to DDSCAT we used the refractory indices of (Draine \& Lee 1984; Laor \& Draine 1993; Weingartner \& Draine 2000). Dust grains with sizes with radii larger than $a>2 \mu \mathrm{m}$ are beyond the reach of DDSCAT. In order to overcome the numerical limit, we combined existing data from DDSCAT with data obtained by the MIEX code (Wolf \& Voshchinnikov 2004) using Mie scattering to smoothly extrapolate our dust model to an upper cutoff radius of $a_{\max }=200 \mu \mathrm{m}$. We consider the upper radius in our outflow 
environment to be larger than in the interstellar medium (ISM) and set the model with $a_{\max }=2 \mu \mathrm{m}$ as our default mode.

The POLARIS RT simulations are performed with the cross sections for a dust grain of average size with

$\bar{C}_{\mathrm{X}}=\sum_{i} \kappa_{i} \cdot \int_{a_{\min }}^{a_{\max }} \pi a^{2}\left(Q_{\mathrm{X}, i, \|}+Q_{\mathrm{X}, i, \perp}\right) n(a) R(a) \mathrm{d} a$,

where $\pi a^{2}$ is the geometrical cross section, $\kappa_{i}$ is the fraction of distinct dust grain materials, and $\bar{C}_{\mathrm{X}}$ stands for the cross sections of extinction $\left(C_{\mathrm{e}}\right)$, absorption $\left(C_{\mathrm{a}}\right)$, and scattering $\left(C_{\mathrm{s}}\right)$, respectively. The same averaging is applied for the cross sections $\Delta C_{\mathrm{e}}$ for dichroic extinction, for thermal re-emission $\Delta C_{\mathrm{a}}$, and circular polarization $\Delta C_{\mathrm{c}}$ with

$\Delta \bar{C}_{\mathrm{X}}=\sum_{i} \kappa_{i} \cdot \int_{a_{\min }}^{a_{\max }} \pi a^{2}\left(Q_{\mathrm{X}, \mathrm{i}, \|}-Q_{\mathrm{X}, \mathrm{i}, \perp}\right) \sin ^{2}(\vartheta) n(a) R(a) \mathrm{d} a$.

Here, the cross sections are weighted by the Rayleigh reduction factor $R(a)$ (see e.g., Greenberg 1968; Lazarian 2007, for details) to account for imperfect grain alignment. $R(a)=1$ corresponds to perfect alignment along the direction of the magnetic field, and $R(a)=0$ to randomly orientated dust grains. The angle $\vartheta$ is defined by the direction of the incident light and the magnetic field direction. Consequently, no linear polarization emerges along a LOS parallel to the magnetic field direction since the dust grain would appear to be spherical (see Reissl et al. 2014, for details).

\section{Dust grain alignment}

The alignment of the rotation axis of a dust grain parallel to the direction of the magnetic field lines is due to paramagnetic effects within the grain material itself (e.g., Davis \& Greenstein 1951; Barnett 1917). However, in the ISM, perfect alignment is suppressed by gas-dust collisions and the interaction with the local radiation field.

Here, we go beyond previous approaches in this field (e.g., Kim \& Martin 1995; Draine \& Fraisse 2009; Padovani et al. 2012) and include the classical imperfect Davis-Greenstein (IDG) alignment that is due to paramagnetic relaxation (Davis \& Greenstein 1951; Jones \& Spitzer 1967; Purcell 1979) with the radiative torque alignment (RAT) that is due to radiation-dust interaction (Dolginov \& Mitrofanov 1976; Draine \& Weingartner 1996, 1997; Lazarian \& Hoang 2007; Hoang \& Lazarian 2009) in the MC RT simulations. Additionally, we consider the randomization of dust grains caused by thermal fluctuations in the dust grain material for the grain alignment efficiency (see Lazarian \& Roberge 1997).

The IDG alignment is mainly determined by the parameter

$\delta_{0}=2.07 \times 10^{20} \frac{\boldsymbol{B}^{2}}{n_{\mathrm{g}} T_{\mathrm{d}} \sqrt{T_{\mathrm{g}}}}$,

which represents an upper threshold for the dust grain alignment. The IDG accounts for the alignment of small dust grains because grains with an effective radius above $\delta_{0}$ do no longer significantly contribute to the net polarization (see Jones \& Spitzer 1967, for details). Ferromagnetic inclusions can enhance the grain alignment efficiency by several orders of magnitude (see, e.g., Andersson et al. 2015, for review).

Irregular dust grains are expected to scatter left-handed and right-handed circular light differently (Dolginov \& Mitrofanov 1976; Weingartner \& Draine 2003). This additional radiative torque (RAT) increases the aliment efficiency (Lazarian \& Hoang 2007; Hoang \& Lazarian 2008). The RAT alignment assumes dust grains to align efficiently when the angular velocity $\omega_{\text {rad }}$ resulting from RATs dominantes the angular velocity $\omega_{\text {gas }}$ caused by random gas bombardment so that $\omega_{\text {rad }} \geq 3 \times \omega_{\text {gas }}$. Consequently, the RAT alignment is determined by the ratio of angular velocities. The minimum grain radius $a_{\mathrm{alg}}$ at which dust grains start to align is determined by

$$
\left(\frac{\omega_{\mathrm{rad}}}{\omega_{\mathrm{gas}}}\right)^{2}=\frac{a_{\mathrm{alg}} \rho_{\mathrm{d}}}{\delta m_{\mathrm{H}}}\left(\frac{t_{\mathrm{gas}}}{\left(t_{\mathrm{gas}}+t_{\mathrm{rad}}\right) n_{\mathrm{g}} k_{B} T_{\mathrm{g}}} \int Q_{\Gamma}(\epsilon) \lambda \gamma_{\lambda} \bar{u}_{\lambda} \mathrm{d} \lambda\right)^{2} .
$$

Here, $\rho_{\mathrm{d}}$ is the density of the dust grain material and $\bar{u}_{\lambda}$ is the local mean energy density of the radiation field. The wavelength specific anisotropy factor $\gamma_{\lambda}$ varies between $\gamma_{\lambda}=1$ for unidirectional radiation and $\gamma_{\lambda}=0$ for an isotropic radiation field. For details about the constant $\delta$ and the characteristic gas drag time $t_{\text {gas }}$ and thermal emission drag time $t_{\text {rad }}$, we refer to Draine \& Weingartner (1997). The radiative torque efficiency depends $Q_{\Gamma}(\epsilon)$ on the angle $\epsilon$ between the predominant direction of radiation and the magnetic field direction and allows calculating the characteristic dust grain size $a_{\mathrm{alg}}$ at which dust grains start to align. For further details see Reissl et al. (2016).

\section{Radiative transfer calculations}

To create synthetic polarization maps, we postprocessed the data resulting from the MHD simulations discussed in Sect. 2 in a three-step approach. First, we updated the initial MHD dust temperature in a Monte Carlo (MC) simulation (see Lucy 1999; Bjorkman \& Wood 2001; Reissl et al. 2016, for details) using the luminosities and position of the emerged cluster of protostars (see Sect. 2).

Second, the anisotropy parameter $\gamma_{\lambda}$ as well as the local energy density required by the RAT alignment was calculated in a second MC process. Here, we again used the method proposed in Lucy (1999), which employs the path lengths and direction of all photons crossing a cell to obtain

$\boldsymbol{u}_{\lambda} \mathrm{d} \lambda=\frac{\epsilon_{0}}{c \Delta t V_{\text {cell }}} \sum_{\mathrm{d} \lambda} \frac{l \cdot \boldsymbol{k}}{|\boldsymbol{k}|}$.

With this, we can finally calculate the polarization maps. The wavelength regime considered in this paper covers the mid-IR to the far-IR, submm and $\mathrm{mm}(\approx 20-3000 \mu \mathrm{m})$.

A polarization pattern can also emerge because of scattering on dust grains. The quantity that quantifies the influence of scattering to the net polarization is the albedo $\alpha=C_{\text {sca }} / C_{\text {ext }} \in[0 ; 1]$, where $\alpha=1$ means that the polarization is completely dominated by scattering and $\alpha=0$ stands for no scattering at all. For the considered dust grain models with upper cutoff radii of $a_{\max }=2 \mathrm{~mm}$, the albedo $\alpha \approx 0.25$ at a wavelength of $\lambda=20 \mu \mathrm{m}$. Hence, scattering can influence the polarization pattern especially near the disk region under such conditions. However, we started our investigation with an upper cut of radius of $a_{\max }=250 \mathrm{~nm}$, where $\alpha \approx 0.01$ at $\lambda=20 \mu \mathrm{m}$. Larger dust grains are only applied for synthetic polarization maps in the $\mathrm{mm}$ regime of wavelength where $\alpha \ll 10^{-3}$ for all dust models. We therefore neglect the influence of scattering in this paper.

The usual way to represent the resulting polarization are the Stokes parameters $(I, Q, U, V)$. Here, $I$ stands for intensity, $Q$ and $U$ for linear polarization, and $V$ for circular polarization. The radiative transfer in the Stokes formalism leads to a set of equations (see Martin 1974, for details) considering the dust grains 


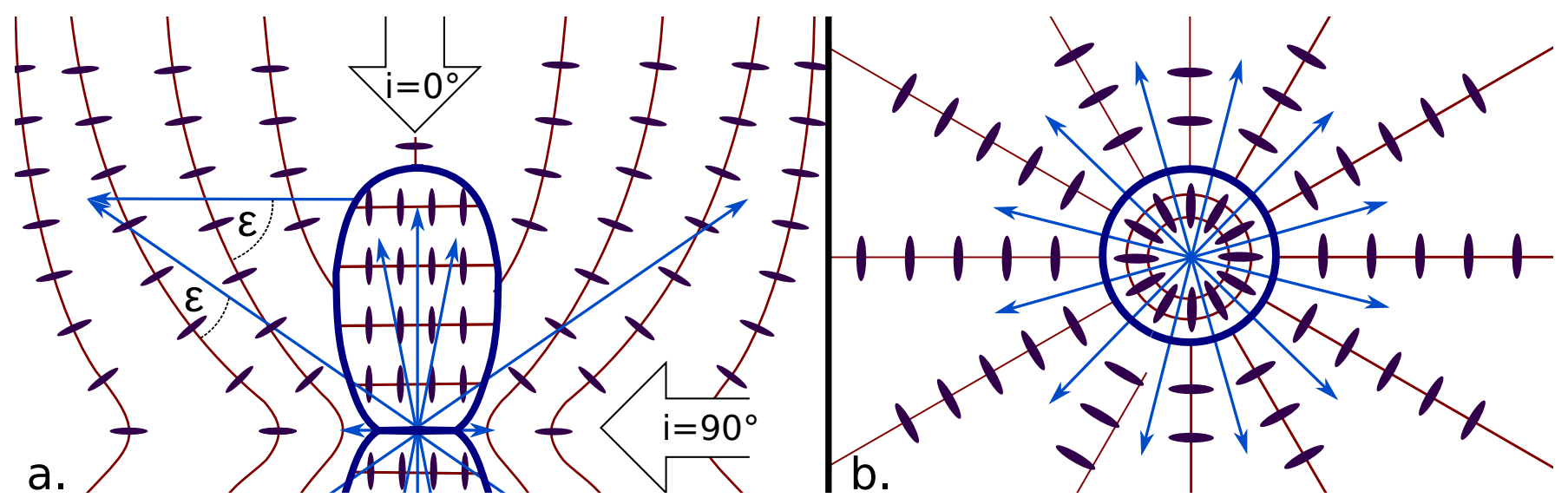

Fig. 2. Schematic illustration of the expected dust grain (dark blue ellipses) alignment behavior according to IDG and RAT alignment in the center plane perpendicular (panel a)) and parallel (panel b)) to the symmetry axis of the outflow lobes (blue). The angle $\epsilon$ is defined to be between the predominant direction of the radiation (light blue arrows) and the magnetic field lines (red). The white arrows indicate the definition of the inclination angle.

as blackbody radiators that can be solved analytically. This allows calculating the contribution $\left(I^{\prime}, Q^{\prime}, U^{\prime}, V^{\prime}\right)$ of each cell (see Whitney \& Wolff 2002) along each path element $\mathrm{d} l$ with

$$
\begin{aligned}
I^{\prime}= & (I+Q) \mathrm{e}^{-n_{\mathrm{d}} l\left(C_{\mathrm{e}}+\Delta C_{\mathrm{e}}\right)}+n_{\mathrm{d}} l B_{\lambda}\left(T_{\mathrm{d}}\right)\left[C_{\mathrm{a}}+\Delta C_{\mathrm{a}} \cos (2 \phi)\right], \\
Q^{\prime}= & (I-Q) \mathrm{e}^{n_{\mathrm{d}} l\left(\Delta C_{\mathrm{e}}-C_{\mathrm{e}}\right)}-n_{\mathrm{d}} l B_{\lambda}\left(T_{\mathrm{d}}\right)\left[C_{\mathrm{a}}+\Delta C_{\mathrm{a}} \cos (2 \phi)\right], \\
U^{\prime}= & \mathrm{e}^{-n_{\mathrm{d}} l C_{\mathrm{e}}}\left[U \cos \left(n_{\mathrm{d}} l \Delta C_{\mathrm{c}}\right)-V \sin \left(n_{\mathrm{d}} l \Delta C_{\mathrm{c}}\right)\right] \\
& +n_{\mathrm{d}} l \Delta C_{\mathrm{a}} B_{\lambda}\left(T_{\mathrm{d}}\right) \sin (2 \phi),
\end{aligned}
$$

and

$$
V^{\prime}=\mathrm{e}^{-n_{\mathrm{d}} l C_{\mathrm{e}}}\left[U \sin \left(n_{\mathrm{d}} l \Delta C_{\mathrm{c}}\right)-V \cos \left(n_{\mathrm{d}} l \Delta C_{\mathrm{c}}\right)\right]
$$

Here, $n_{\mathrm{d}}$ is the number density of the dust and $B_{\lambda}\left(T_{\mathrm{d}}\right)$ is the Planck function. The angle $\phi$ is measured between the direction of light polarization and the magnetic field lines. We note that because of the $\phi$ dependency, circular polarization can only emerge in regions where each following magnetic field line and subsequently the preferential axis of grain alignment is non-parallel to the previous line along the LOS (see, e.g., Martin 1974). The hourglass component in the outside region is such a magnetic field with rather parallel field lines along the LOS. As a result of this, we obtain most of the circular polarization from within the outflow lobes in our RT calculations. Here, the maximum of the degree of circular polarization can amount to up to $\pm 0.4 \%$. As shown in Reissl et al. (2014), the pattern of circular polarization provides additional information to distinguish between different well-ordered magnetic field morphologies. However, because of the chaotic nature of the helical field, the results are inconclusive and do not allow us to identify the helical component by any characteristic circular polarization pattern. We therefore focus on linear polarization in this paper. However, circular polarization still needs to be considered in the RT calculation because of the permanent transfer from circular polarization ( $V$-parameter) to linear polarization ( $U$-parameter) and vice versa.

The degree and orientation of linear polarization $P_{1}$ is determined by

$P_{1}=\frac{I_{\mathrm{p}}}{I}=\frac{\sqrt{Q^{2}+U^{2}}}{I}$,

where $I_{\mathrm{p}}$ is the polarized intensity. Its position $\chi_{\mathrm{Pl}}$ angle on the plane of the sky is defined by

$\chi_{\mathrm{Pl}}=\frac{1}{2} \arctan \left(\frac{U}{Q}\right)$.
For a more detailed description we refer to Reissl et al. (2014, 2016).

\section{Synthetic polarization maps}

\subsection{Linear polarization by RAT and IDG alignment}

In this section we investigate how the polarization pattern depends on grain alignment theory, inclination angle, and wavelength. Here, it is essential to separately estimate the expected contributions of different grain alignment theories to the net polarization. For the physical conditions in the MHD simulation, both IDG and RAT alignment theory predict a similar behavior with regard to the preferential axis of grain alignment. However, the alignment efficiency and consequently the detectability of a polarization signal may differ significantly for different alignment theories. Furthermore, the two competing polarization mechanisms of dichroic extinction and thermal re-emission both contribute to linear polarization. Dichroic extinction dominates polarization in the UV, optical, and near-IR regime and leads to polarization parallel to the magnetic field direction. In contrast to dichroic extinction, thermal reemission results in light polarization perpendicular to the magnetic field for a wavelength from the mid-IR to the $\mathrm{mm}$. It is expected that in an intermediate regime of wavelengths, the two effects cancel each other out and that therefore the polarization vectors flip their orientation by $90^{\circ}$ in the transition. In order to determine the intermediate regime of wavelengths where dichroic extinction transitions to thermal reemission, we therefore simulated synthetic polarization maps. The maps were calculated with an upper cutoff radius of $a_{\max }=2 \mu \mathrm{m}$ and cover a wavelength regime of $20 \mu \mathrm{m}-3 \mathrm{~mm}$. The disk is seen edge-on.

In Fig. 2 an illustration of the expected grain alignment is shown for a better interpretation of the following polarization maps. For an inclination of $90^{\circ}$ the resulting maps of linear polarization calculated with IDG and RAT alignment theory are shown in Fig. 3. Although the IDG and RAT alignment theories are based on different physical principles, the resulting overall orientation of polarization vectors over wavelength are comparable. However, we note that the degree of linear polarization varies significantly between the two alignment theories, with RAT leading to much higher polarization values.

With increasing wavelength, the contribution of thermal reemission to polarization increasingly dominates dichroic 

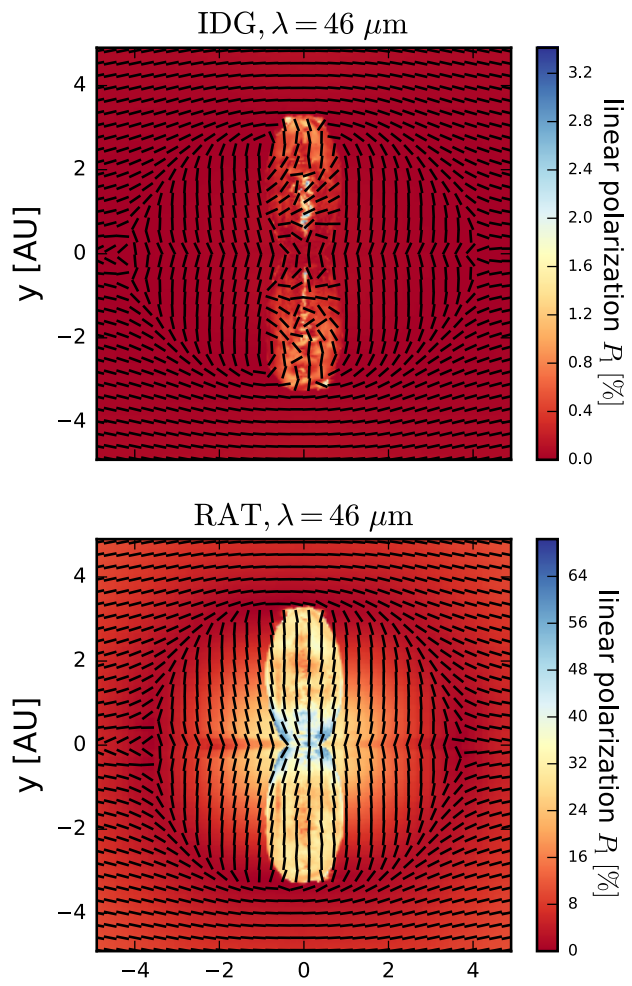

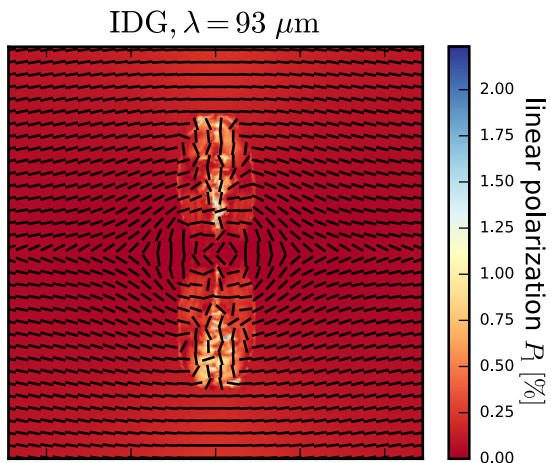

RAT, $\lambda=93 \mu \mathrm{m}$

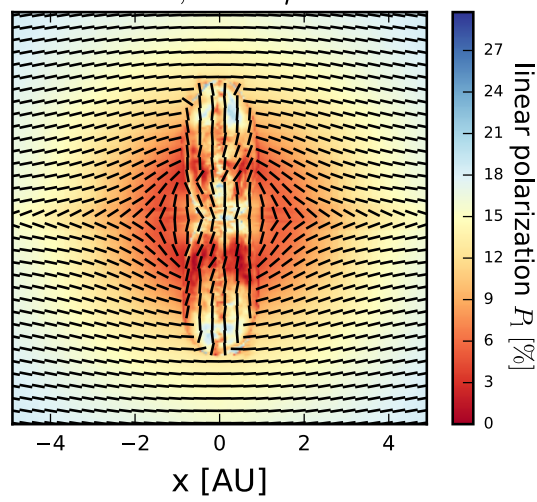

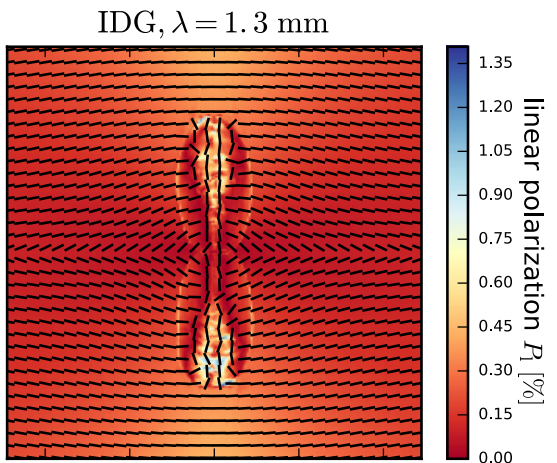

$\operatorname{RAT}, \lambda=1.3 \mathrm{~mm}$

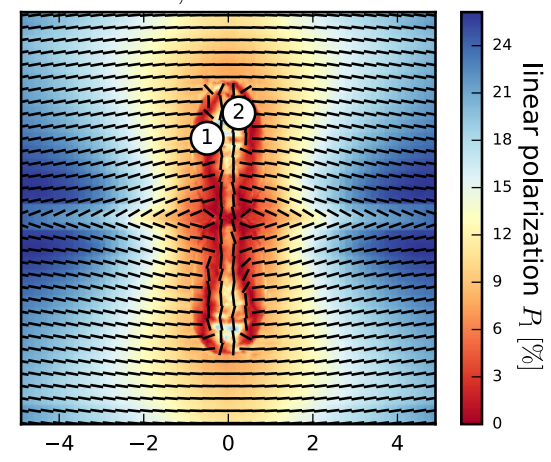

Fig. 3. Maps showing the degree of linear polarization $P_{1}$ (color-coded) overlaid with normalized orientation vectors for the three distinct wavelengths of $\lambda=46 \mu \mathrm{m}$ (left column), $\lambda=93 \mu \mathrm{m}$ (middle column), and $\lambda=1.3 \mathrm{~mm}$ (right column) at an inclination angle of $90^{\circ}$. The top row shows the maps considering IDG alignment, while the bottom row shows the results considering RAT alignment. The numbers in the right bottom panel correspond to the LOS shown in Figs. 5 and 6. Only the panels for $\lambda=93 \mu \mathrm{m}$ trace the helical magnetic field.

extinction. Since the two competing polarization effects contribute in directions perpendicular to each other, it is possible that the net polarization vector flips by $90^{\circ}$ or is even canceled out.

The wavelength at which thermal reemission starts to appear in the polarization maps for both IDG and RAT alignment is at $\lambda \simeq 40 \mu \mathrm{m}$. The outermost regions of the polarization maps are less dense than the center and hence are less strongly affected by dichroic extinction. Therefore, polarization by thermal reemission appears first in the outer part of the polarization maps an moves toward the center for longer wavelength. For a wavelength of $\lambda \simeq 46 \mu \mathrm{m}$, the outflow lobe is still unaffected by the effect of flipping polarization vectors (Fig. 3 left column). Here, the outer regions of the polarization maps are dominated by thermal reemission, while the center regions are still polarized as a result of dichroic extinction. In the case of RAT alignment (Fig. 3 left bottom panel), this leads to a characteristic ring-shaped gap with a minimum degree of linear polarization where the contributions of dichroic extinction and thermal reemission cancel each other out. For the IDG alignment (Fig. 3 left top panel), this effect is less pronounced and hardly detectable because the degree of linear polarization in that area is generally low. The IDG alignment is highly suppressed in high-density and hightemperature regions (see Eq. (3)) and would thus allow no conclusion about the underlying magnetic field morphology near the disk region.

At a wavelength of about $\lambda \simeq 93 \mu \mathrm{m}$ (see Fig. 3 middle column), the outflow lobes are completely enclosed by the region of thermal reemission, while the polarization in the lobes itself results from dichroic extinction. This is due to the dust temperature of $T_{\mathrm{d}} \approx 30-40 \mathrm{~K}$ at the surface of the outflow lobes (see
Fig. 1 middle panel), which corresponds to a peak emission at $\lambda \approx 70-97 \mu \mathrm{m}$. The outside regions with $T_{\mathrm{d}} \approx 10 \mathrm{~K}$ contribute a neglectable amount of radiation at that wavelength regime. Consequently, the dust grains in front of the outflow lobes are illuminated by a strong background radiation emitted by the surface of the outflows, and dichroic extinction dominates the polarization.

At even longer wavelengths for both RAT and IDG, thermal dust reemission dominates the entire map at $\lambda \simeq 600 \mu \mathrm{m}$, resulting in a polarization pattern comparable to that shown in Fig. 3 in the right columns. For a longer wavelength, the polarization vectors are perpendicular to the projected magnetic field and their orientation remains rather constant up to the mm wavelength regime.

As shown by the detailed analysis (see Sect. 7), even in the inner disk region, the linear polarization is completely due to thermal remission. Hence, in the maps considering IDG and RAT alignment, the orientation of the polarization vectors represent the projected magnetic field morphology. In contrast to RAT alignment, the helical component remains slightly more apparent at the tops and near the symmetry axis of the outflow lobes for IDG.

\subsection{Effect of inclination angle}

So far, we have examined the polarization orientation and degree dependence on grain alignment theory and wavelength for a fixed inclination angle of $90^{\circ}$ between the outflow axis and the LOS. However, interpretations of polarization measurements are also influenced by projection effects. In this section we to investigate how the linear polarization pattern changes as a function of the inclination toward the observer. Owing to the 

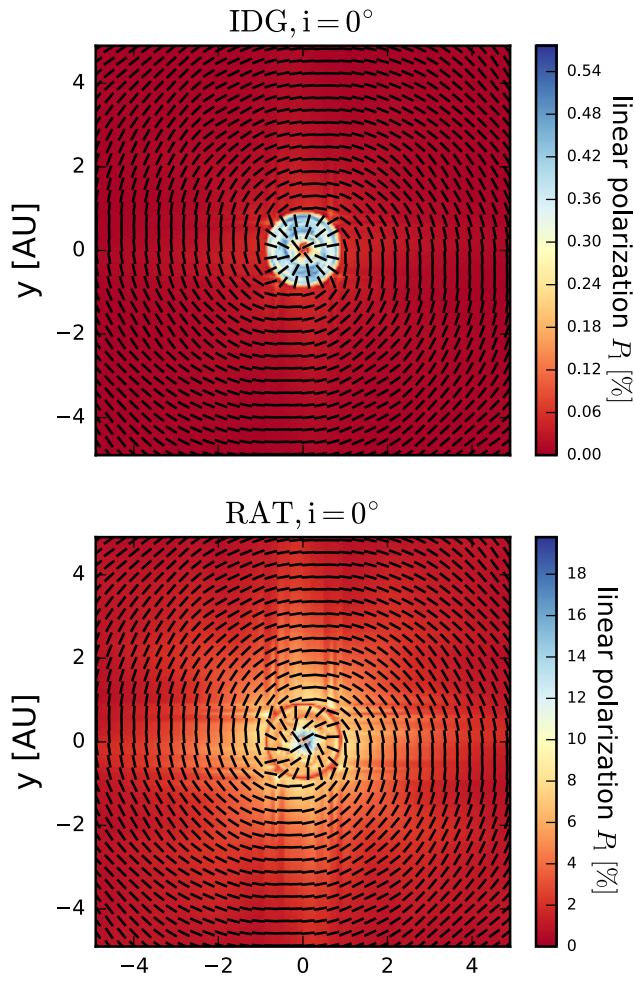

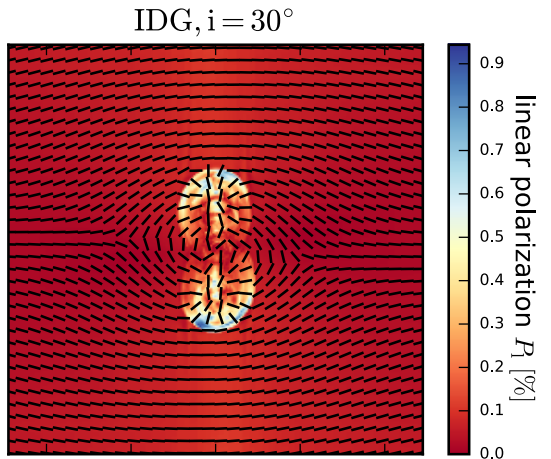

$\mathrm{RAT}, \mathrm{i}=30^{\circ}$

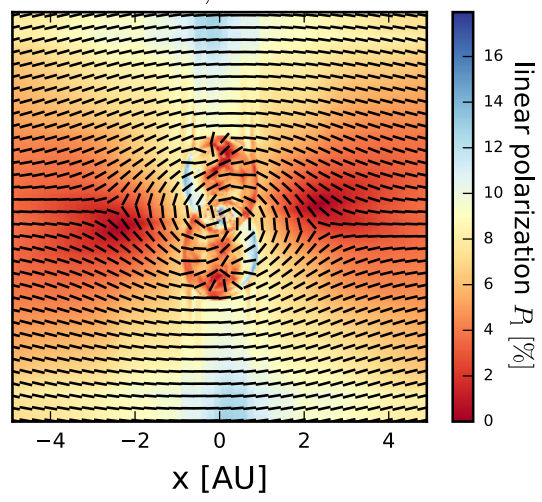

$\mathrm{IDG}, \mathrm{i}=60^{\circ}$

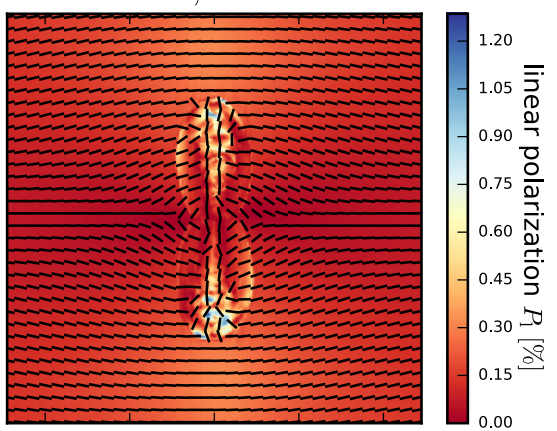

$\operatorname{RAT}, \mathrm{i}=60^{\circ}$

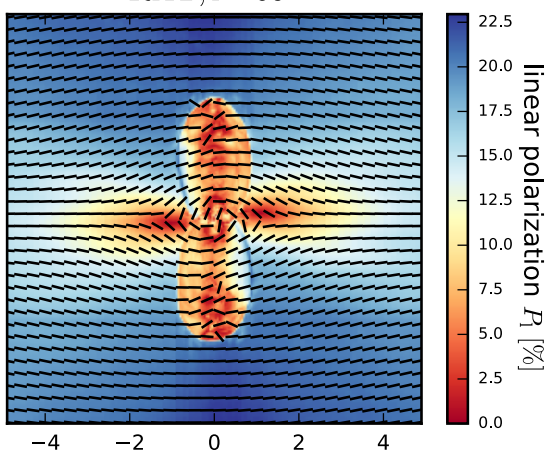

Fig. 4. Maps showing the degree of linear polarization $P_{1}$ (color-coded) overlaid with normalized orientation vectors for a wavelength of $\lambda=$ $1.3 \mathrm{~mm}$ at the three distinct inclination angles $i=0^{\circ}$ (left column), $i=30^{\circ}, i=0^{\circ}$ (middle column), and $i=60^{\circ}$ (right column). The top row shows the maps considering IDG alignment, while the bottom row shows the results considering RAT alignment. Note also that the polarization degree varies strongly between the two alignment theories and for different wavelengths.

ambiguities in the mid-IR and submm discussed in Sect. 6.1 and with regard to the simulation of synthetic observations with the ALMA telescope array, we focus here on a wavelength of $\lambda=1.3 \mathrm{~mm}$, where the polarization is purely due to thermal reemission, meaning that the polarization vector is perpendicular to the field.

Figure 4 shows polarization maps considering IDG alignment in comparison to RAT alignment for the three different inclination angles $i$ of $0^{\circ}$ (disk is face-on), $45^{\circ}$, and $90^{\circ}$, respectively. Again, we note that the polarization degree differs by more than one order of magnitude. For an IDG alignment and an inclination angle of $i=0^{\circ}$ (Fig. 4 top left panel), the LOS toward the center of the maps is parallel to the hourglass magnetic field (see Fig. 2). Since no linear polarization can occur in the case of a LOS parallel to the magnetic field (see Sect. 3), the linear polarization emerges completely in the interior of the outflow lobes and the disk component. With increasing inclination angle, the contributions from the hourglass field start to dominate the overall orientation of linear polarization. For an inclination of $i=45^{\circ}$ (see middle top panel of Fig. 4), the polarization pattern begins to resemble the projected hourglass morphology. The helical component of the magnetic field, however, remains apparent close to the symmetry axis and at the tips of the outflows, meaning that the orientation of the polarization vectors is predominantly vertical.

In the bottom row of Fig. 4 we show the linear polarization maps considering RAT alignment. For an inclination angle of $i=0^{\circ}$ the results are qualitatively the same as those for IDG alignment. However, with increasing inclination angle the contributions of the surrounding hourglass-shaped magnetic field dominate earlier than for IDG alignment. Here, only the regions close to tips of the outflow lobes match the helical magnetic field component, meaning that the polarization vectors are vertically oriented.

When the subsequent magnetic field lines cross each other along the LOS, the polarized emission of a given dust grain is canceled out by the emission of another one, leading to an area of reduced linear polarization. This projection effect of crossing field lines along the LOS becomes apparent in the maps with RAT alignment (Fig. 4 bottom row) where two polarization holes (bottom middle panel) and two additional lobes of minimum linear polarization (bottom right panel) become visible perpendicular to the symmetry axis of the outflow lobes. Here, these additional lobes are not a result of reduced dust density or temperature fluctuations, but are simply a projection effect and an indicator of the underlying hourglass-shaped field morphology (see also Reissl et al. 2014).

The same effect can be observed for the polarization maps with IDG alignment in the top middle panel and top right panel of Fig. 4. However, the already low degree of linear polarization due to inefficient grain alignment outside the outflow lobes makes these projection effects less relevant.

Despite the additional ferromagnetic inclusions considered for the IDG alignment calculations, the maximum degree of linear polarization is on the order of a few per cent. This makes RAT alignment the relevant alignment process for observations in the presented outflow environment. Hence, recent efforts of Hoang \& Lazarian (2016) showed that it is possible to combine IDG and RAT alignment. However, we conclude that IDG is negligible in the considered outflow environment and focus on RAT alignment alone in the following sections. 

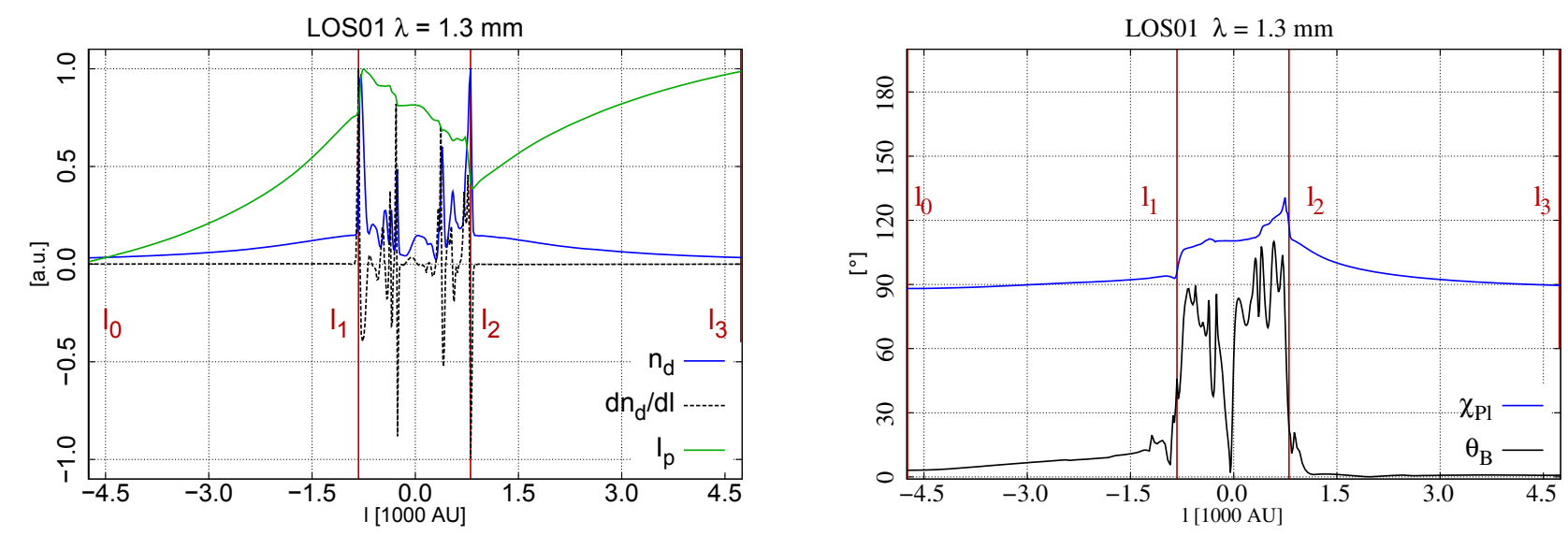

Fig. 5. Physical quantities along the a exemplary LOS \#1 in the bottom right of Fig. 3 . The vertical red lines indicate the borders between the first outer region $\left(l_{0}-l_{1}\right)$, the interior of the outflow lobes $\left(l_{1}-l_{2}\right)$, and the second outer region $\left(l_{2}-l_{3}\right)$. Left panel: normalized values of polarized intensity $I_{\mathrm{p}}$ (green), dust number density $n_{\mathrm{d}}$ (blue), and its first derivative $\mathrm{d} n_{\mathrm{d}}(l) / \mathrm{d} l$ (dotted black). The polarized intensity $I_{\mathrm{p}}$ is plotted as a cumulative value. Right panel: orientation angles of linear polarization $\chi_{\mathrm{P}_{1}}$ (blue) and magnetic field direction $\theta_{\mathrm{B}}$ (black). An orientation angle of $0^{\circ}$ corresponds to vertical polarization vectors in all figures shown in this paper.
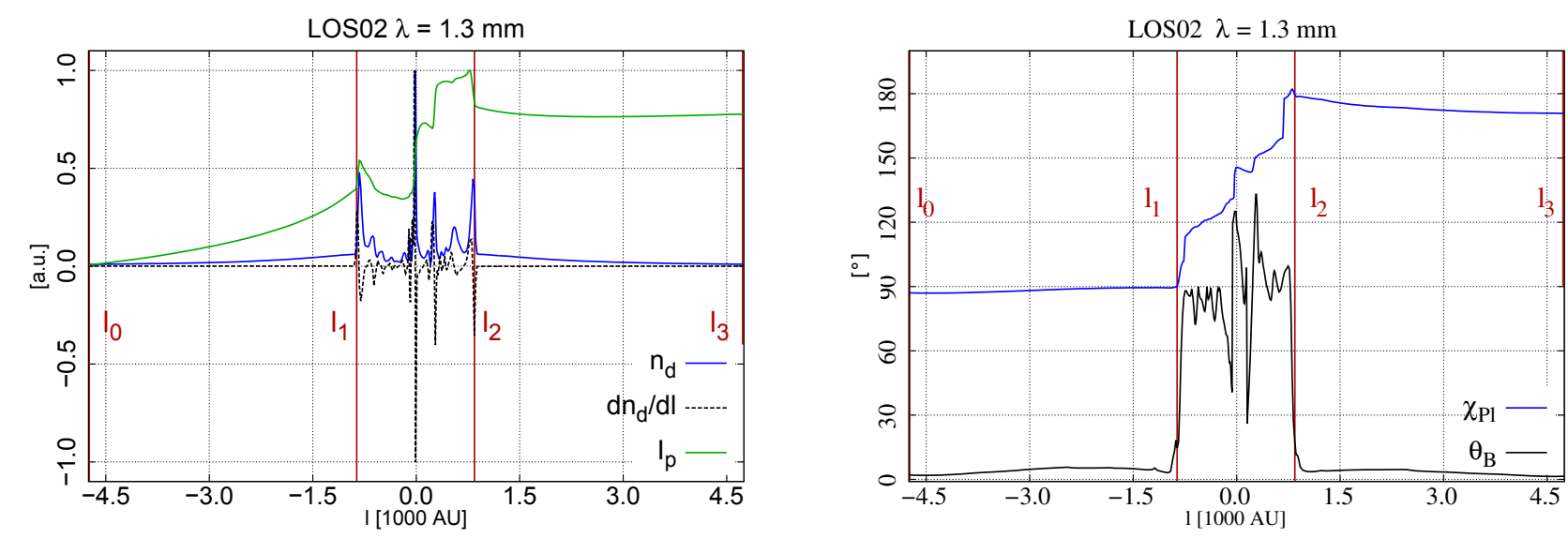

Fig. 6. Same as Fig. 5 along the LOS \#2 in the bottom right panel of Fig. 3.

\section{Origin of linear polarization}

In the previous sections we discussed maps of linear polarization on the basis of physically well-motivated dust grain alignment theories and dust modeling. In these efforts it remained unclear to what extend the outflow lobes and the surrounding medium contribute to the synthetic maps of net polarization. Consequently, the synthetic polarization maps of Figs. 3 and 4 still remain ambiguous regarding the question of what component of the magnetic field is traced (hourglass in front of the outflow lobes or in the helical field).

This problem is even more severe for actual observations. Here, the spatial information of density and temperature as well as the magnetic field morphology in any observed astrophysical system are lost as a result of the projection along a particular LOS. In contrast to observations, synthetic data by RT calculations allow tracing different LOS through the 3D MHD simulation and subsequently to determine the origin of polarization. In the following we focus on the origin and the detectability of the polarization pattern characteristic for helical and hourglass magnetic field structures. To this end, we implemented a heuristic algorithm to analyze the polarization state of radiation along a distinct LOS $l$.

This automatized heuristic approach works in three steps:

1. Identification of the distinct regions inside and outside the outflow lobes. Here, we detect the bow shock of the outflow lobes by analyzing the first derivative of the gas number density $\mathrm{d} n_{\mathrm{g}} / \mathrm{d} l$ (Figs. 5 and 6 left panels, the black dotted lines). This allows us to distinguish between three regions along the LOS. The first outside region expands from $l_{0}$ to $l_{1}$, the outflows itself from $l_{1}$ to $l_{2}$, and the second outside region from $l_{2}$ to $l_{3}$ (plotted in Figs. 5 and 6 as red vertical lines).

2. Ray-tracing through the MHD simulation data in order to keep track of the accumulated polarized intensity $I_{\mathrm{p}}$ (Figs. 5 and 6 left panels, the green lines), the orientation angle of linear polarization $\chi_{\mathrm{Pl}}$ (Figs. 5 and 6 right panels, the blue lines), and the orientation angle $\theta_{\mathrm{B}}$ (Figs. 5 and 6 right panels, the black lines) of the magnetic field with respect to the symmetry axis of the outflow lobes.

3. Determining the origin of polarization by analyzing the largest increase in $I_{\mathrm{p}}$ as well as the relative orientation between magnetic field lines and linear polarization.

With this simple but effective scheme, the origin of linear polarization can be determined. The first criterion is the increase or decrease of polarized intensity $I_{\mathrm{p}}$ along each LOS. Here, it is sufficient to compare the accumulated polarized intensity at the points $I_{p}\left(l_{0}\right), I_{p}\left(l_{1}\right), I_{p}\left(l_{2}\right)$, and $I_{p}\left(l_{3}\right)$ to determine the area with the largest increase.

The second criterion is the resulting polarization angle with respect to the local magnetic field direction. Although the magnetic field direction in the outflow lobes is not well ordered, it appears indeed rather regular in projection and can therefore be 

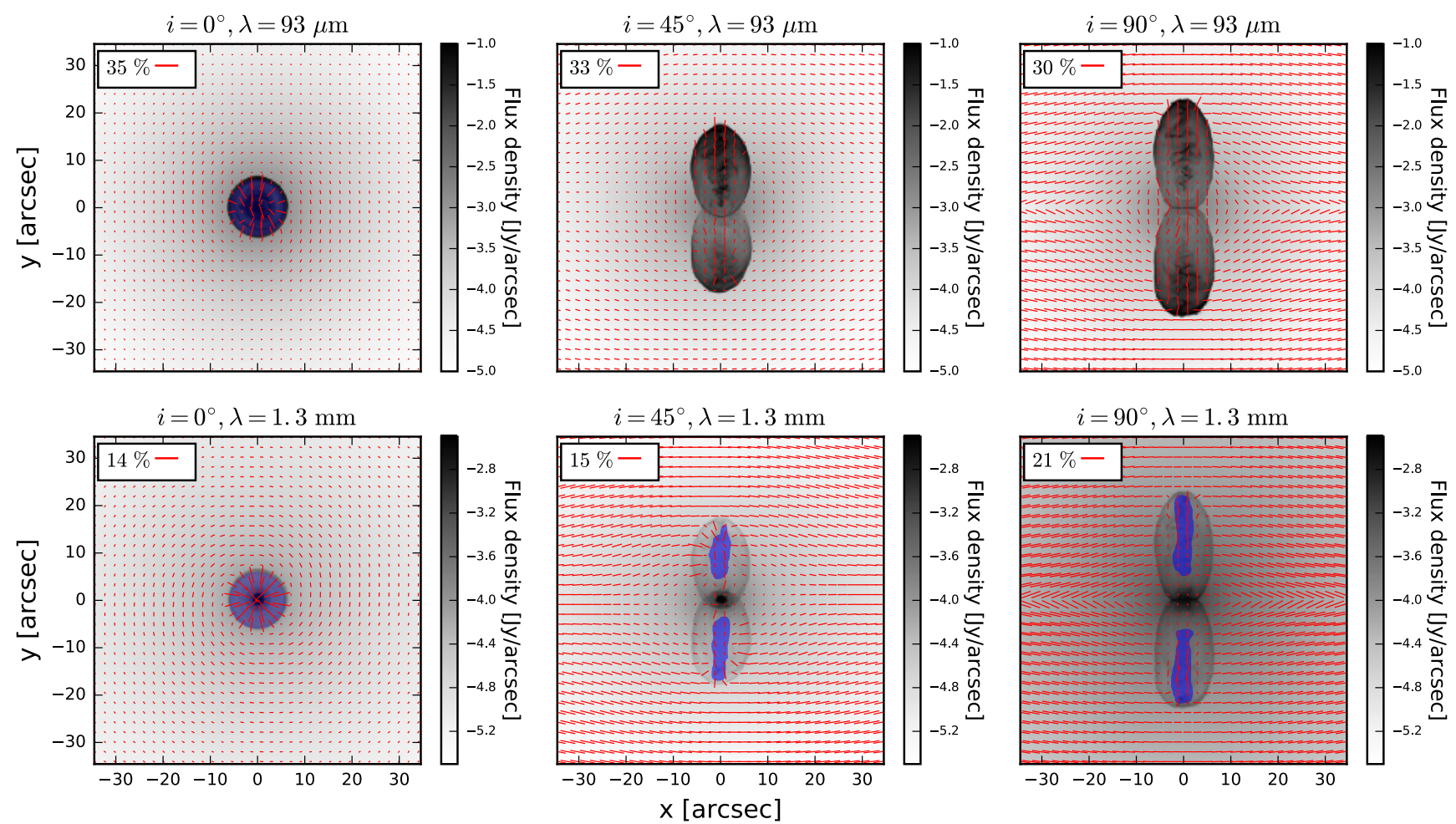

Fig. 7. Synthetic flux density maps (gray coded) with a wavelength of $\lambda=93 \mu \mathrm{m}$ (top row) and $\lambda=1.3 \mathrm{~mm}$ (bottom row) and a distance of $140 \mathrm{pc}$ overlaid with vectors of linear polarization considering RAT alignment for inclination angles of $i=0^{\circ}$ (left column), $i=45^{\circ}$ (middle column), and $i=90^{\circ}$ (right column) and a maximal dust grain radius of $a_{\max }=2 \mu \mathrm{m}$. The length of the vectors depends on the degree of linear polarization. For better comparison the color bar is fixed in each row. The blue areas indicate where the polarization vectors represent the helical magnetic field structure of the outflow according to the LOS analysis.

assumed to be perpendicular to the projected magnetic field direction in both outside regions.

We assume that the linear polarization originates from the interior of the outflow lobes when the largest increase in polarized intensity is inside the outflow lobe, and the orientation vector of linear polarization deviates from the projected helical field direction by less than $\pm 20^{\circ}$.

Here it needs to be emphasized that this heuristic method is fine-tuned to probe the outflow lobes in just this particular MHD simulation. The parameters of this heuristic approach are optimized by proper testing by minimizing the false-positive results. A manual evaluation of 75 randomly chosen LOSs for each of the different inclination angles and wavelengths revealed that the accuracy of the correct detection of the origin of linear polarization is better than $85 \%$. Consequently, the areas in the polarization maps where linear polarization originates from the inside of the outflow lobes can be identified with high precision. However, the number of LOSs probing the helical field might be larger because of possible false-negative detections.

Figures 5 and 6 show the resulting plots of two exemplary LOSs corresponding to the positions in the right bottom panel of Fig. 3 at a wavelength of $\lambda=1.3 \mathrm{~mm}$. In the left panel of Fig. 5 the polarized intensity $I_{\mathrm{p}}$ emerges in the first outer region $\left(l_{0}-l_{1}\right)$ and jumps to a maximum near the edge at $l_{1}$ of the outflow lobe, decreases in the interior $\left(l_{1}-l_{2}\right)$ with a strong correlation to jumps in dust number density $n_{\mathrm{d}}$ and reaches its absolute maximum at the border of the grid at position $l_{3}$.

In the first outside region $\left(l_{0}-l_{1}\right)$ the orientation angle $\chi_{\mathrm{Pl}}$ (see Eq. 11) remains at an almost constant value of roughly $90^{\circ}$ with respect to the magnetic field orientation $\theta_{B}$ and increases slightly near the first edge $\left(l_{1}\right)$ of the outflow lobe, as is shown in the right panel of Fig. 5. In the interior $\left(l_{1}-l_{2}\right)$ the polarization angle again remains almost constant and trends back to $90^{\circ}$ as the radiation propagates toward the border $\left(l_{3}\right)$ of the model space. In this case, the interior of the outflow lobe is of minor influence to linear polarization with respect to degree and orientation. Clearly, the LOS01 does not probe the helical component of the magnetic field morphology, but only the hourglass foreground.

Along the second LOS shown in Fig. 6, the polarized intensity $I_{\mathrm{p}}$ shows the same trend as in Fig. 5 and the orientation angle $\chi_{\mathrm{Pl}}$ remains at around $90^{\circ}$ up to the point of $l_{1}$ in the first outside region. However, in the center of the outflow lobe the accumulated amount of $I_{\mathrm{p}}$ rises to a global maximum and remains almost constant throughout the second outer region $\left(l_{2}-l_{3}\right)$. In contrast to LOS01, the orientation of linear polarization rotates by $90^{\circ}$ between $l_{1}$ and $l_{2}$. Here, the linear polarization matches the helical field component. In this case, the resulting linear polarization probes the interior of the outflows and hence the helical magnetic field component.

The different polarization behavior between LOS01 and LOSO2 may possibly be attributed to a more chaotic and accordingly less clearly pronounced helical field component close to the borders of the outflow lobes. Furthermore, the gas temperature $T_{\mathrm{g}}$ is higher at the border. The RAT alignment efficiency is inversely proportional $T_{\mathrm{g}}$ (see Eq. (4)). This may also contribute to the difference along LOS01 and LOS02.

Figure 7 shows intenssity maps overlaid with vectors of linear polarization and different inclination angles for $\lambda=93 \mu \mathrm{m}$ in comparison with maps for $\lambda=1.3 \mathrm{~mm}$. Here, we assumed only RAT alignment and a typical distance of a nearby star-forming region 140 pc (e.g., Preibisch \& Smith 1997; Torres et al. 2007; Mamajek 2008). Hence, the maps have a field of view of 69.1 arcmin with a resolution of $1024 \times 1024$ px corresponding to $0.068 \times 0.068$ arcsec. Areas with a positive detection of the 
helical magnetic field component according to the LOS analysis presented in this section are marked in blue.

For the maps with $\lambda=93 \mu \mathrm{m}$ (Fig. 7 top row) the linear polarization traces the helical field component only for an inclination angle of $i=0^{\circ}$. The reason is that linear polarization cannot emerge when the LOS is parallel to the magnetic field direction (see Sect. 3). This means that the flip of the polarization vectors by $90^{\circ}$ for an inclination of $i=45^{\circ}$ and $i=90^{\circ}$ does not arise because we probe the inner helical field. We rather observe the outer hourglass field in extinction, whereas in the surrounding it is probed in reemission. Consequently, there are no areas marked blue in these panels.

In contrast to the maps with $\lambda=93 \mu \mathrm{m}$, in the synthetic observations at $\lambda=1.3 \mathrm{~mm}$ (Fig. 7 bottom row) the polarization pattern is due to thermal reemission. Thus, any polarization vector flip by $90^{\circ}$ is an indicator only of a different magnetic field morphology. The LOS analysis confirms that the helical magnetic field morphology of the outflow can be well traced in the outflow center, quite independently of inclination angle.

The contribution of the disk region to the maps shown in Fig. 7 is minuscule. For an inclination of $i=90^{\circ}$ the height of the disk is below the assumed resolution. With decreasing inclination, the projected area of the disk increases. However, as the LOS analysis reveals, the polarization is completely dominated by either the outflows itself or the outside regions, but not for the disk. Hence, probing the magnetic field morphology in the disk itself seems to be impossible for the chosen MHD simulation and within the selected set of parameters presented in this paper.

\section{Grain size dependency}

Dust grains in the ISM are very likely to be of submicron size (see Sect. 3). In contrast to this, grains inside protoplanetary disks and outflows can differ significantly in size from grains in the diffuse ISM. Grain growth in the disk is expected to enrich the surrounding environment with dust grains of sizes up to $\lesssim 1 \mathrm{~mm}$ (e.g., Sahai et al. 2006; Hirashita \& Li 2013). The choice of upper dust grain size may significantly affect the resulting synthetic intensity and polarization maps and subsequently the predictions for future observational missions. Hence, in this section we investigate the effect of the inclination angle together with the effect of the upper dust grain size on intensity and linear polarization.

The sizes of dust grains are most probably not evenly distributed in all regions. Larger dust grains coagulating in the disk are expected to be blown out along the outflow lobes. Near the tip of the outflow lobes, the dust grain sizes are reprocessed toward smaller radii because of the high temperature and pressure in this area. This complexity is beyond the scope of the current analysis, and we therefore follow a simpler approach with a constant upper cutoff radius of $a_{\max }$ throughout the model space. We precalculated dust cross sections for two additional dust models with distinct cutoff radii of $a_{\max }=250 \mathrm{~nm}$ in accordance with the standard MRN model (see Sect. 3) and an extreme case with $a_{\max }=200 \mu \mathrm{m}$. We repeated the postprocessing of the MHD data for the RT pipeline as described in Sect. 5.

Figure 8 shows the resulting intensity map at a wavelength of $1.3 \mathrm{~mm}$ overlaid with the vectors of linear polarization dependent on inclination and the different dust grain models. As for Fig. 7, we assumed a distance of $140 \mathrm{pc}$ to the outflow system. Areas with a positive detection of the helical magnetic field component according to the LOS analysis presented in the previous section are marked in blue. These findings agree independently of the applied dust grain model or inclination because only the regions close to the symmetry axis of the outflow lobes are accessible by observations. The same applies for the orientation of linear polarization. The pattern of the polarization vectors shown in Fig. 8 (see Fig. 7 bottom row) is in good agreement with the results presented in Sect. 6.2. The overall polarization pattern calculated with an upper dust grain size of $a_{\max }=250 \mathrm{~nm}$ (Fig. 8 top row) and $a_{\max }=200 \mu \mathrm{m}$ (Fig. 8 bottom row) are rather similar to each other. There are differences regarding intensity and degree of linear polarization. In contrast to the other maps with $a_{\max }=250 \mathrm{~nm}$, synthetic images with an upper dust grain size of $a_{\max }=200 \mu \mathrm{m}$ (Fig. 8 right column) show a higher intensity with a reduced degree of linear polarization. However, the finding that the helical magnetic field component within the outflow lobes should in principle be detectable for a wavelength of $\lambda=1.3 \mu \mathrm{m}$ holds independently of the applied dust grain model (see also Fig. 7).

\section{Synthetic observations}

In contrast to the ideal scenarios of the prevision sections, realistic observing conditions considering instrumental and atmospheric effects may further complicate the interpretation of polarization data. In this section we translate our synthetic intensity and polarization data into observational maps. Here the focus is on determining whether the areas where the helical field dominates linear polarization presented in Sects. 7 and 8 would still be detectable by observations.

With regard to the limitations of wavelength without a flip of polarization vectors (see Sect. 6.1) as well as the expected field of view (see Fig. 7 bottom row) of the post-processed MHD outflow simulation, the ALMA (Brown et al. 2004) observatory provides the necessary equipment to constrain the observable parameter of linear polarization, and subsequently, that of the underlying magnetic field morphology. We calculated polarization and intensity data for three typical wavelength of $850 \mu \mathrm{m}, 1.3 \mathrm{~mm}$, and $3.0 \mathrm{~mm}$ for dust grains with an upper cutoff of radius of $a_{\max }=2 \mu \mathrm{m}$ of the size distribution. We assumed a mosaic observation with an object-observer distance of $140 \mathrm{pc}$. The Stokes $I, Q$, and $U$ maps were separately processed, making use of the standard ALMA reduction software CASA (McMullin et al. 2007) with the simobserve and clean task. Here, we applied a resolution of 1 arcsec, an observation time of five hours, and we included thermal noise as well as precipitable water vapor of $0.5 \mathrm{~mm}$ to mimic the atmosphere.

Initially, the observability of the outflow lobes in the resulting $I, Q$, and $U$ maps was heavily disturbed by the very bright inner disk regions because of the dominating influence of the overall PSF. Since these regions are very compact, most of the emission is in the longest baseline. Hence, we limited the visibility (in the uv-plane) in order to take care of these bright disk regions. Finally, we combined the $I, Q$, and $U$ maps to create synthetic maps of intensity and linear polarization with Eqs. (10) and (11).

Figure 9 shows the resulting flux density maps overlaid with the scaled vectors of linear polarization for our simulated ALMA data. In the synthetic observations with a wavelength of $\lambda=850 \mu \mathrm{m}$ (Fig. 9 left column), the detectable intensity only covers the disk region and the very edges of the outflow lobes. This is related to the point-spread function (PSF), dominated by the brightest contributions located in the inner disk and the followup cleaning process (see McMullin et al. 2007). However, these detectable regions still coincide with the regions 

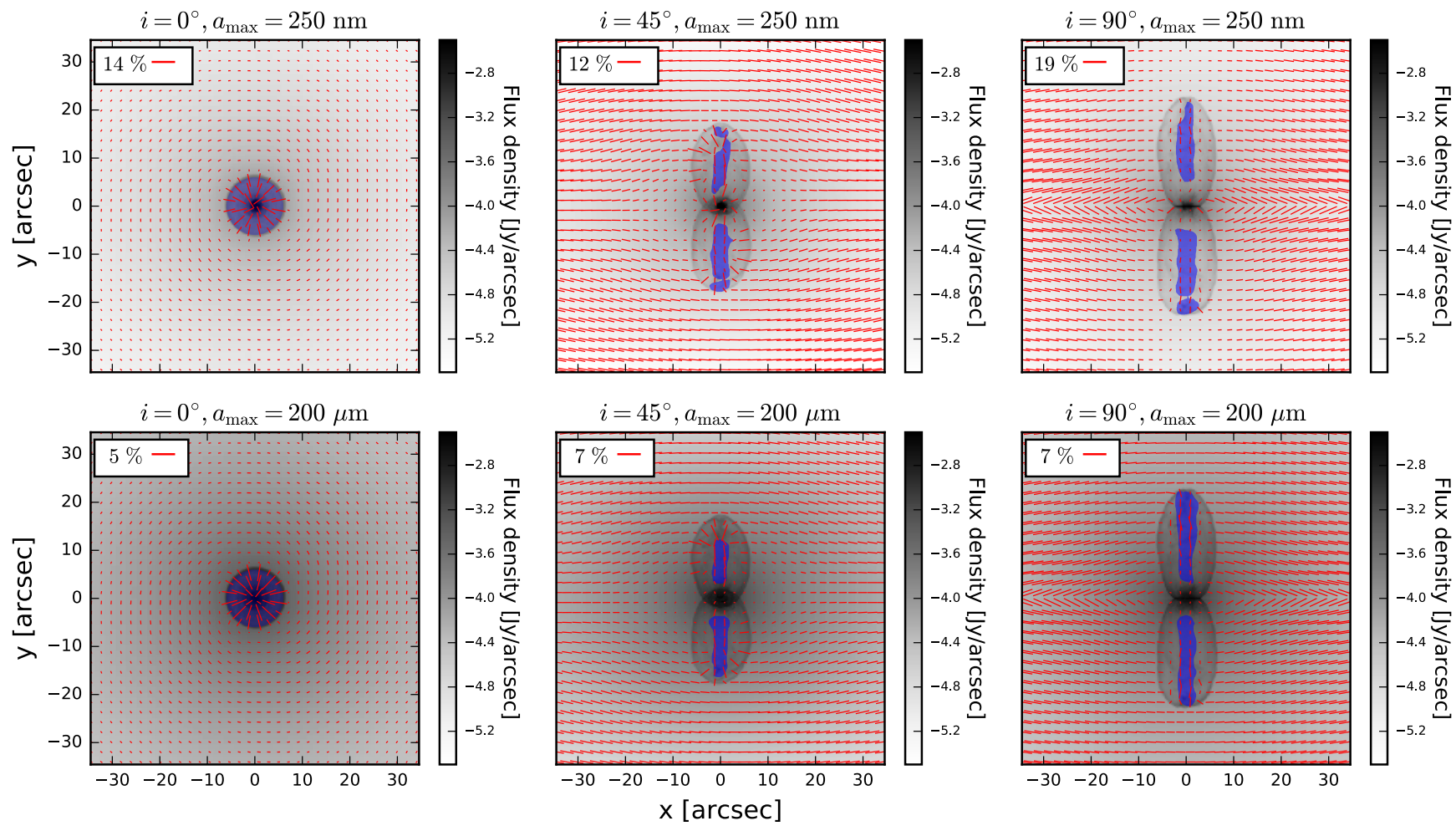

Fig. 8. Synthetic flux density maps (gray coded) with a wavelength of $\lambda=1.3 \mathrm{~mm}$ and a distance of 140 pc overlaid with vectors of linear polarization considering RAT alignment for inclination angles of $i=0^{\circ}$ (left column), $i=45^{\circ}$ (middle column), and $i=90^{\circ}$ (right column) and maximum dust grain radii of $a_{\max }=250 \mathrm{~nm}$ (top row) and $a_{\max }=200 \mu \mathrm{m}$ (bottom row). The length of the vectors depends on the degree of linear polarization. For better comparison the color bar is fixed between $F_{\min }=-4.0 \log _{10}(\mathrm{Jy} / \operatorname{arcsec})$ and $F_{\max }=-2.0 \log (\mathrm{Jy} / \operatorname{arcsec})$ in all plots. The blue areas indicate where the polarization vectors represent the helical magnetic field morphology of the outflow itself according to the LOS analysis.

determined with the LOS analysis (see Sect. 7). Although the ALMA instrument seems to be barely suitable to detect a polarization signal emerging from our post-processed MHD simulation at $\lambda=850 \mu \mathrm{m}$, it is in principle possible to detect a polarization signal from within the outflow lobe since the detectability increases even further for even longer wavelengths. At a wavelength of $\lambda=1.3 \mathrm{~mm}$ (Fig. 9 middle column) the interior of the outflow lobe and subsequently the helical magnetic field morphology is partially observable for low inclination angles. Even with an increase in inclination angle of up to $90^{\circ}$ the helical field would still be partly accessible by polarization measurements with ALMA. For $\lambda=3.0 \mathrm{~mm}$ (see Fig. 9 right column) the observable intensity completely covers the outflow lobes. The only exception is an inclination angle of $90^{\circ}$. Here, the blue area of the LOS analysis is is marginally larger then the intensity observable with ALMA. However, as Fig. 9 shows in the right bottom panel, a wavelength of $3.0 \mathrm{~mm}$ provides the optimal configuration to distinguish the helical magnetic field morphology embedded in the outflow lobes from the surrounding hourglass field. This is because the largest area found with the LOS analysis coincides with intensity and the polarization pattern is minimally influenced by the PSF in the outflow lobes.

\section{Discussion}

\subsection{Dichroic extinction versus thermal reemission}

The examination of multiwavelength polarization measurements highlights one of the main obstacles for the interpretation of dust polarization measurements. The polarization effects of dichroic extinction and thermal reemission contribute simultaneously to linear polarization with the preferential polarization axes perpendicular to each other. Both contributions to polarization can be calculated exactly (see Sect. 5). Here, the most relevant parameters are the cross section of dichroic extinction $\Delta C_{\mathrm{e}}$ and absorption $\Delta C_{\mathrm{a}}$. In the applied dust model of Sect. 3, the cross $\Delta C_{\mathrm{e}}$ dominates toward shorter wavelengths, while for absorption $\Delta C_{\mathrm{a}}$ increases with wavelength. We can therefore expect a flip of $90^{\circ}$ toward longer wavelengths.

Dust temperature and number density also vary along the LOS, and different regions of the system dominate the overall polarization calculation as a function of wavelength, resulting in an additional offset of the projected magnetic field direction. As a result of this, a characteristic area where the two effects cancel each other out is visible as a clear ring structure of minimum polarization, shown in the left column of Fig. 3. Polarization maps of that type are prone to be misinterpretated. Several physical effects such as regions with a reduced dust density, a large amount of crossing magnetic field lines along the LOS, or a randomization of dust grain orientation by a high-velocity stream (e.g., Rao et al. 1998) may also account for a reduction in the degree of linear polarization.

An even more ambiguous polarization pattern represents the result shown in the middle column of Figs. 3 and 7. A helical magnetic field morphology in the interior of the outflow lobe is expected from theoretical predictions. Hence, by comparing the alignment behavior shown in Fig. 2 with the orientation vectors of linear polarization in the middle panels of Figs. 3, we might easily conclude that we probe the distinct helical field of the overall magnetic field morphology. However, the LOS analysis introduced in Sect. 7 reveals a different picture. The particular polarization map at a wavelength of $\lambda=93 \mu \mathrm{m}$ only matches the hourglass component of the magnetic field 

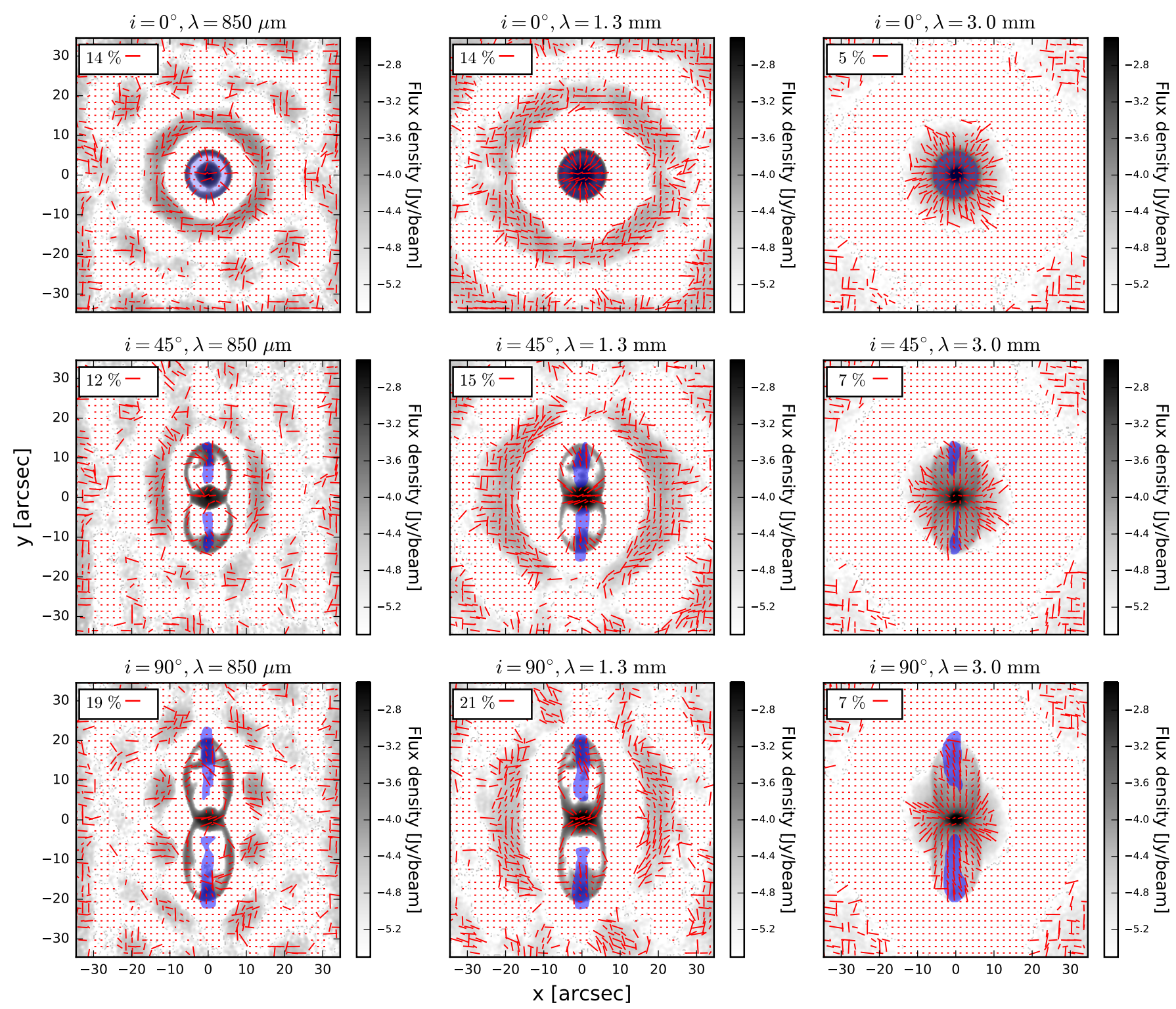

Fig. 9. Synthetic ALMA flux density maps (gray coded) for a wavelength of $\lambda=850 \mu \mathrm{m}$ (left column), $\lambda=1.3 \mathrm{~mm}$ (middle column), and $\lambda=3.0 \mathrm{~mm}$ (right column) at a distance of $140 \mathrm{pc}$ overlaid with vectors of linear polarization considering RAT alignment for inclination angles of $i=0^{\circ}$ (top row), $i=30^{\circ}$ (middle row), and $i=60^{\circ}$ (bottom row). The length of the vectors depends on the degree of linear polarization. For better comparison the color bar is fixed between $F_{\min }=-4.0 \log _{10}(\mathrm{Jy} /$ beam $)$ and $F_{\max }=-2.0 \log _{10}(\mathrm{Jy} / \mathrm{beam})$ in all plots. The blue areas indicate where the helical magnetic field morphology is potentially detectable according to the LOS analysis.

morphology throughout the polarization map as a result of two different polarization effects. In the outer parts it is thermal reemission, while close to the outflow lobes the polarization vectors switch by $90^{\circ}$ as a result of dichroic extinction. The helical magnetic field morphology cannot be traced up to a wavelength of about $\lambda \gtrsim 600 \mu \mathrm{m}$.

These ambiguities become irrelevant with decreasing inclination angles. A polarization signal does not emerge from aligned dust grains when the LOS and the magnetic field direction are parallel (see Eq. (2)). Consequently, the hourglass field cannot contribute to linear polarization with a LOS along the outflow direction (compare Fig. 2a). Hence, for low inclination angles the helical morphology of the magnetic field is not hidden by the hourglass component and can be identified unambiguously.

Chapman et al. (2013) investigated the correlation between the direction of the magnetic field lines in low-mass cores and the bipolar outflows. The direction and degree of linear polarization observed is in good agreement with the polarization in presented in Sect. 6.1. Especially the decreasing degree of linear polarization toward the outflow (see Figs. 7 and 8) is consistent with our result. This confirms the validity of the approach of creating synthetic polarization maps by combining dust continuum RT with dust grain alignment theories.

\subsection{Alignment-specific polarization pattern}

The RT simulations reveal a marginal contribution of IDG alignment to the net polarization, making RAT clearly the dominant alignment mechanism in this outflow scenario. Since RAT alignment also depends on the alignment efficiency $Q_{\Gamma}(\epsilon)$ (see Eq. (4)), where $\epsilon$ is the angle between radiation field and magnetic field, we could also expect to detect this characteristic in the resulting polarization pattern (see Andersson et al. 2011; Reissl et al. 2016). However, in the post-processing of the MHD 
simulation such an angle-dependent effect is not noticeable because of the local dust temperature distribution and its contribution to the overall radiation field. In contrast to a point-like source, the heated and dense surface of the outflow lobes acts like a light bulb that illuminates the model space from more than one direction (compare Fig. 2 left panel) and the radiation field is more diffuse than it would be for a point-like source. Hence the angle-dependency of $Q_{\Gamma}(\epsilon)$ becomes less relevant.

\subsection{Role of dust grain size}

For the maps in Fig. 8 (upper row) we used the standard MRN model with a cutoff of $a_{\max }=250 \mathrm{~nm}$. In this model RT calculations showed that the maximum size of RAT alignment (see Sect. 5) exceeded the upper cut of radius in a considerable amount of regions in the outflow simulation $\left(a_{\mathrm{alg}} \geq a_{\mathrm{max}}\right)$. We might therefore expect a lower degree of linear polarization. However, because of the lack of larger dust grains, the model with $a_{\max }=250 \mathrm{~nm}$ ( 8 top row) is optically thinner than other models with larger cutoff radii. This results in weaker extinction and consequently flux, and the polarization remains similar to the model with $a_{\max }=2 \mu \mathrm{m}$ shown in Fig. 7 (bottom row). The model with $a_{\max }=200 \mu \mathrm{m}$ is shown in Fig 8 (bottom row). Dust grains of the largest size are quite rare in the dust mixture because of the standard MRN power-law size distribution (see Sect. 3). However, the higher reemission cross section $C_{\mathrm{a}}$ of larger dust grains at mm wavelengths compensates for their lower abundance. This results in a higher flux for the model with $a_{\max }=200 \mu \mathrm{m}$ than in the other models by a factor of $\approx 2$. Additionally, with increasing cutoff radii the polarization along the LOS becomes also rapidly dominated by larger dust grains. However, in the range of wavelength where $\lambda \approx a$, the cross section of reemitted polarization is smallest. Consequently, the peak value of linear polarization is reduced for a model with $a_{\max }=200 \mu \mathrm{m}$ by a factor of between 2 and 3 .

\subsection{Constraints to observational equipment}

The synthetic polarization maps show that the helical field morphology in the interior of the outflow lobes is not easily accessible by polarization measurements with aligned dust grains (see Figs. 7 and 8). This holds even more reconsidering the limitations of the observational equipment. The instrument $H A W C+$ mounted on the airborne telescope S OFIA (Dowell et al. 2013) is capable of linear polarization measurements. However, its field of view (between $2.7 \times 1.7 \mathrm{arcmin}$ and $8.0 \times 6.1 \mathrm{arcmin}$ ) and spectral coverage $(53-214 \mu \mathrm{m})$ means that it is not suitable for observing the particular outflow scenario presented in this paper (see also Sect. 10). Additionally, the instrument limit of polarized intensity does not allow probing the interior of the outflow. In order to use the full field of view in the available $H A W C+$ bands, our protostellar outflow object should also be at a distance between $10 \mathrm{pc}$ and $40 \mathrm{pc}$. However, there is no star-forming region within such a distance (Preibisch \& Smith 1997). Furthermore, as shown in Sect. 6.1, the regime of wavelengths where the transition of dominant polarization mechanism from dichroic extinction to thermal re-emission takes place coincides with the $H A W C+$ bands. Consequently, the measurements with the $H A W C+$ instrument would be inconclusive with regard to the traced magnetic field direction. We performed an additional LOS analysis for all $H A W C+$ bands that revealed that the observed polarization pattern would completely represent the projected outside hourglass magnetic field morphology. The helical field inside the outflow lobe cannot be detected within the $H A W C+$ bands. The detectability of the helical magnetic field morphology is only given in the far-IR, submm, and $\mathrm{mm}$ wavelength regime (see Sect.7).

In contrast to $S O P H I A / H A W C+$, the ALMA telescope can probe the interior of the outflow lobes especially in the submm and $\mathrm{mm}$ regime. Here, the limitations lie in the influence of the brightest regions in the disk regions and their influence on the observability of the outflow lobes. While the outside regions cannot completely be covered even at a wavelength of $\lambda=3.0 \mathrm{~mm}$, the polarization of the outflow lobe, especially the polarization emerging from the helical magnetic field component, is accessible by ALMA for that wavelength. However, we used an ideal non-turbulent MHD simulation for the RT calculations. Although ALMA observations can probe the interior of the outflow lobes, this task may be challenged in more realistic environments with additional blending by turbulent motions.

\section{Summary and conclusions}

We presented synthetic polarization maps from the mid-IR to the mm wavelength regime of a post-processed MHD protostellar outflow simulation. The post-processing was performed with the RT code POLARIS (Reissl et al. 2016). Here, we considered different grain alignment theories, inclination angles, and dust models in order to constrain the parameters that allow detecting the helical magnetic field component in the outflow lobes that are embedded in a larger hourglass-shaped field of the surrounding medium.

The conclusions of this study are as follows:

1. With increasing wavelength the transition between dichroic extinction and thermal reemission manifests itself in a flip of the orientation angles of $90^{\circ}$ in linear polarization. Additionally, areas where these transition takes place are depolarized, and the magnetic field morphology is no longer accessible by observations. The polarization maps are completely dominated by thermal reemission at $\lambda \simeq 600 \mu \mathrm{m}$ and the orientation in polarization pattern remains fixed. The low polarization degree in the outflows is in accordance with the findings of Tomisaka (2011) and Chapman et al. (2013).

2. We developed a heuristic method to identify the origin of the polarization. We showed that the helical magnetic field structure inside the outflow lobe is observable only close to the symmetry axis of the lobe and at the tip of the outflow. Outside these regions, the polarisation emerges from the hourglass magnetic field structure in the foreground of the outflow.

3. The alignment of dust grains does not result in polarization when the LOS is parallel to the magnetic field direction. This effect is independent of the considered dust grain alignment theory and wavelength. However, this fact is of advantage in the particular case of the post-processed molecular outflows MHD simulation because it allows us to probe the interior of the outflow lobes for low inclination angles.

4. Synthetic polarization maps were calculated considering different grain alignment theories. The polarization is dominated by RAT alignment that produces polarization degrees of a few percent up to $\sim 10 \%$ in agreement with observations. In contrast, the IDG alignment does not produce measurable polarization degrees. This holds only for the investigated MHD simulation and we caution that this might not be true in general.

5. Probing the interior of the outflow lobes depends on the maximum size of the dust grain distribution. We simulated 
polarization map with a power-law size distribution considering different upper grain sizes. A composition with larger grains leads to a higher intensity, but also to a lower polarization and vice versa. However, the overall pattern of linear polarization seems to be independent of the cutoff radius. We expect the best observability for an upper cutoff in the order of $\approx 1 \mu \mathrm{m}$.

6. From the observational point of view, the best conditions to probe the interior of the outflow lobes is under inclination angles close to $0^{\circ}$ or $90^{\circ}$ for a wavelengths from the far-IR $(\lambda \gtrsim 600 \mu \mathrm{m})$ to the $\mathrm{mm}$ regime.

7. The interior of the outflow, that is, the helical field structure, cannot be probed with SOPHIA/HAWC+, since in the available bands the polarization is dominated by the hourglass field in the foreground of the outflows.

8. In contrast, ALMA observations should potentially allow probing even the interior of the outflow lobes and subsequently to distinguish between the helical magnetic field in the outflow and the larger hourglass-shaped field structure in the surrounding medium.

As shown in this paper, the origin of the polarization remains ambiguous at best and cannot be easily inferred from observations alone. However, progress is possible by creating physically well-motivated synthetic polarization maps and designing methods of analysis that allow constraining the parameter of possible helical field detection for future observations.

Acknowledgements. We wish to thank the referee for a careful review and for the comments that helped improve our paper. We also wish to thank Gesa H.-M. Bertrang and Robert Brauer for useful discussions about RT and dust grain alignment, and Eric Pellegrini, Álvaro Sánchez-Monge, and Thushara Pillai for their help with simulating synthetic ALMA/CASA data. Also thanks to Stefanie Walch-Gassner for her invitation to Cologne that made this project possible. For this project the authors S.R. and S.W. acknowledge the support of the DFG: WO 857/11-1. D.S. acknowledges funding by the DFG via the Sonderforschungsbereich SFB 956 Conditions and Impact of Star Formation as well as funding by the Bonn-Cologne Graduate School. The MHD simulations were performed at the supercomputer HLRB-II at the Leibniz Rechenzentrum in Garching. We also acknowledge support from the Deutsche Forschungsgemeinschaft in the Collaborative Research Center (SFB 881). The Milky Way System (subprojects B1, B2, and B8) and in the Priority Program SPP 1573 Physics of the Interstellar Medium (grant numbers KL 1358/18.1, KL 1358/19.2). R.S.K. furthermore thanks the European Research Council for funding in the ERC Advanced Grant STARLIGHT (project number 339177).

\section{References}

Andersson, B.-G., Pintado, O., Potter, S. B., Straižys, V., \& Charcos-Llorens, M. 2011, A\&A, 534, A19

Andersson, B.-G., Lazarian, A., \& Vaillancourt, J. E. 2015, ARA\&A, 53, 501

Banerjee, R., Pudritz, R. E., \& Anderson, D. W. 2006, MNRAS, 373, 1091

Barnett, S. J. 1917, Phys. Rev., 10, 7

Belley, F., Ferré, E. C., Martín-Hernández, F., et al. 2009, Earth and Planetary Science Letters, 284, 516

Bjorkman, J. E., \& Wood, K. 2001, ApJ, 554, 615

Blandford, R. D., \& Payne, D. G. 1982, MNRAS, 199, 883

Bouchut, F., Klingenberg, C., \& Waagan, K. 2007, Numerische Mathematik, 108,7

Brown, R. L., Wild, W., \& Cunningham, C. 2004, Adv. Space Res., 34, 555

Chapman, N. L., Davidson, J. A., Goldsmith, P. F., et al. 2013, ApJ, 770, 151

Ching, T.-C., Lai, S.-P., Zhang, Q., et al. 2016, ApJ, 819, 159

Clayton, G. C., Wolff, M. J., Sofia, U. J., Gordon, K. D., \& Misselt, K. A. 2003 ApJ, 588, 871

Crutcher, R. M. 2004, in The Magnetized Interstellar Medium, eds. B. Uyaniker, W. Reich, \& R. Wielebinski, 123

Davidson, J. A., Novak, G., Matthews, T. G., et al. 2011, ApJ, 732, 97

Davis, Jr., L., \& Greenstein, J. L. 1951, ApJ, 114, 206

Djouadi, Z., Gattacceca, J., D'Hendecourt, L., et al. 2007, A\&A, 468, L9

Dolginov, A. Z., \& Mitrofanov, I. G. 1976, Ap\&SS, 43, 291

Dowell, C. D., Staguhn, J., Harper, D. A., et al. 2013, in AAS Meeting Abstracts, $221,345.14$
Draine, B. T., \& Flatau, P. J. 2000, DDSCAT: The discrete dipole approximation for scattering and absorption of light by irregular particles, astrophysics Source Code Library

Draine, B. T., \& Flatau, P. J. 2013, ArXiv e-prints [arXiv: 1305.6497]

Draine, B. T., \& Fraisse, A. A. 2009, ApJ, 696, 1

Draine, B. T., \& Lee, H. M. 1984, ApJ, 285, 89

Draine, B. T., \& Li, A. 2007, ApJ, 657, 810

Draine, B. T., \& Weingartner, J. C. 1996, ApJ, 470, 551

Draine, B. T., \& Weingartner, J. C. 1997, ApJ, 480, 633

Federrath, C., Banerjee, R., Clark, P. C., \& Klessen, R. S. 2010, ApJ, 713, 269

Frau, P., Galli, D., \& Girart, J. M. 2011, A\&A, 535, A44

Fryxell, B., Olson, K., Ricker, P., et al. 2000, ApJS, 131, 273

Girart, J. M., Rao, R., \& Marrone, D. P. 2006, Science, 313, 812

Girart, J. M., Beltrán, M. T., Zhang, Q., Rao, R., \& Estalella, R. 2009, Science, 324, 1408

Girart, J. M., Patel, N., Vlemmings, W. H. T., \& Rao, R. 2012, ApJ, 751, L20

Greenberg, J. M. 1968, Interstellar Grains (The University of Chicago Press), 221

Hall, J. S. 1949, Science, 109, 166

Hildebrand, R. H., \& Dragovan, M. 1995, ApJ, 450, 663

Hildebrand, R. H., Davidson, J. A., Dotson, J. L., et al. 2000, PASP, 112, 1215

Hiltner, W. A. 1949, Nature, 163, 283

Hirashita, H., \& Li, Z.-Y. 2013, MNRAS, 434, L70

Hoang, T., \& Lazarian, A. 2008, MNRAS, 388, 117

Hoang, T., \& Lazarian, A. 2009, ApJ, 697, 1316

Hoang, T., \& Lazarian, A. 2016, ApJ, 831, 159

Hull, C. L. H., Plambeck, R. L., Bolatto, A. D., et al. 2013, ApJ, 768, 159

Hull, C. L. H., Plambeck, R. L., Kwon, W., et al. 2014, ApJS, 213, 13

Jones, R. V., \& Spitzer, Jr., L. 1967, ApJ, 147, 943

Kim, S.-H., \& Martin, P. G. 1995, ApJ, 444, 293

Laor, A., \& Draine, B. T. 1993, ApJ, 402, 441

Lazarian, A. 2007, J. Quant. Spectrosc. Rad. Transf., 106, 225

Lazarian, A., \& Hoang, T. 2007, MNRAS, 378, 910

Lazarian, A., \& Roberge, W. G. 1997, ApJ, 484, 230

Lee, H. M., \& Draine, B. T. 1985, ApJ, 290, 211

Lucy, L. B. 1999, A\&A, 344, 282

Mamajek, E. E. 2008, Astron. Nachr., 329, 10

Martin, P. G. 1971, MNRAS, 153, 279

Martin, P. G. 1974, ApJ, 187, 461

Martin, P. G., \& Angel, J. R. P. 1976, ApJ, 207, 126

Mathis, J. S. 1986, ApJ, 308, 281

Mathis, J. S., Rumpl, W., \& Nordsieck, K. H. 1977, ApJ, 217, 425

McMullin, J. P., Waters, B., Schiebel, D., Young, W., \& Golap, K. 2007, in Astronomical Data Analysis Software and Systems XVI, eds. R. A. Shaw, F. Hill, \& D. J. Bell, ASP Conf. Ser., 376, 127

Offner, S. S. R., Klein, R. I., McKee, C. F., \& Krumholz, M. R. 2009, ApJ, 703, 131

Padovani, M., Brinch, C., Girart, J. M., et al. 2012, A\&A, 543, A16

Preibisch, T., \& Smith, M. D. 1997, A\&A, 322, 825

Pudritz, R. E., \& Norman, C. A. 1983, ApJ, 274, 677

Purcell, E. M. 1979, ApJ, 231, 404

Rao, R., Crutcher, R. M., Plambeck, R. L., \& Wright, M. C. H. 1998, ApJ, 502, L75

Reissl, S., Wolf, S., \& Seifried, D. 2014, A\&A, 566, A65

Reissl, S., Wolf, S., \& Brauer, R. 2016, A\&A, 593, A87

Sahai, R., Young, K., Patel, N. A., Sánchez Contreras, C., \& Morris, M. 2006, ApJ, 653, 1241

Seifried, D., Banerjee, R., Klessen, R. S., Duffin, D., \& Pudritz, R. E. 2011, MNRAS, 417, 1054

Seifried, D., Pudritz, R. E., Banerjee, R., Duffin, D., \& Klessen, R. S. 2012, MNRAS, 422, 347

Shibata, K., \& Uchida, Y. 1985, Astronomical Herald, 78, 240

Soler, J. D., Hennebelle, P., Martin, P. G., et al. 2013, ApJ, 774, 128

Spitzer, L. 1978, Physical processes in the interstellar medium (New York: Wiley-Interscience)

Tomisaka, K. 1998, ApJ, 502, L163

Tomisaka, K. 2011, PASJ, 63, 147

Torres, R. M., Loinard, L., Mioduszewski, A. J., \& Rodríguez, L. F. 2007, ApJ, 671,1813

Weingartner, J. C., \& Draine, B. T. 2000, in BAAS, 32, AAS Meeting Abstracts, 1466

Weingartner, J. C., \& Draine, B. T. 2003, ApJ, 589, 289

Whitney, B. A., \& Wolff, M. J. 2002, ApJ, 574, 205

Wolf, S., \& Voshchinnikov, N. V. 2004, Comput. Phys. Commun., 162, 113

Zhukovska, S., Dobbs, C., Jenkins, E. B., \& Klessen, R. S. 2016, ApJ, 831, 147

Zubko, V. G. 1995, in Wolf-Rayet Stars: Binaries; Colliding Winds; Evolution, eds. K. A. van der Hucht, \& P. M. Williams, IAU Symp., 163, 355 